\title{
توظيف نظرية الصفوف لتعزيز إدارة المعرفة في مرافق المعلومات
}

\author{
عبدالرشـبـد بن عبد العزيز حافظ \\ أستاذ - قسم علم المعلومات بـات \\ جامعة الملك عبدالعزيز - جدة \\ aahafez@kau.edu.sa
}

مستخلص : (1)

تعرض الدراسة واحدة من أهم أدوات تحليل وتصميم نظم المعرفة وهي نظرية الصفوف

تئن Queuing Theory

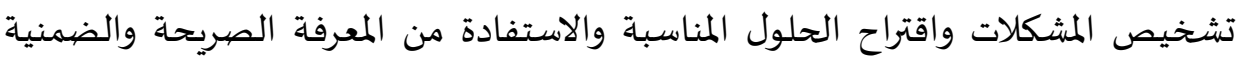

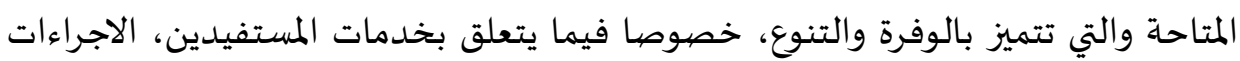

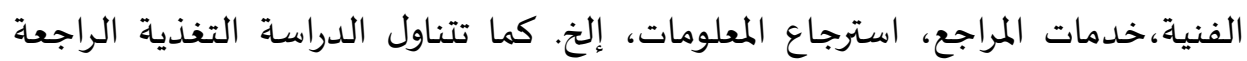

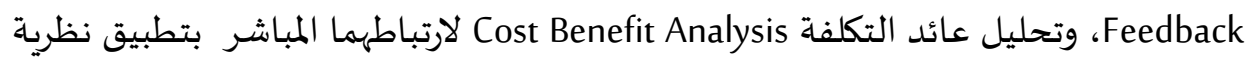
الصفوف. الكلمات المفتاحية :نظرية الصفوف؛ مرافق المعلومات؛ ادارة المعرفة. المعرفة 
نعيش في عصر التسارع التقني، ويشكو الجميع من ضيق الوقت والرغبة في إنجاز أكبر

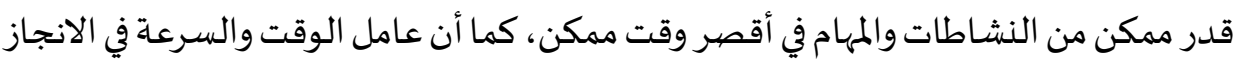

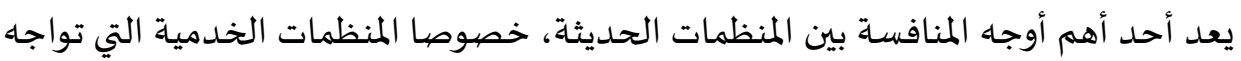

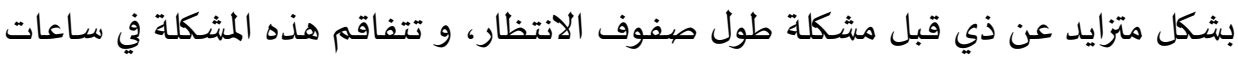

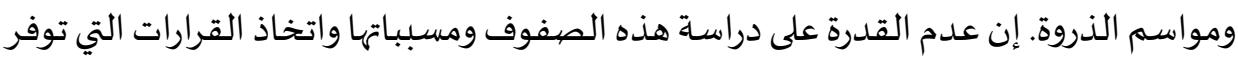

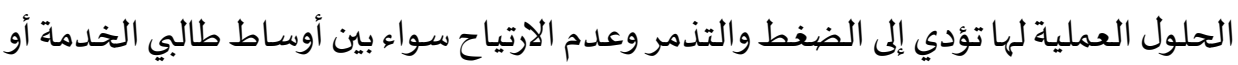
العاملين في مراكز الخدمة، وبطبيعة الحال يؤدي ذلك إلى عدم رضا الطرفين. (1) والمكتبات ومراكز المعلومات شأها شأن أي مرفق حيوي يأخذ بأسباب التطور ويتعامل

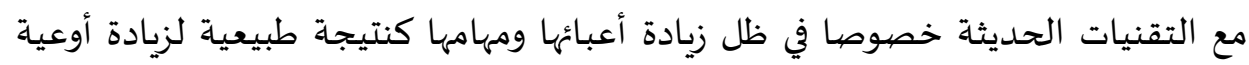

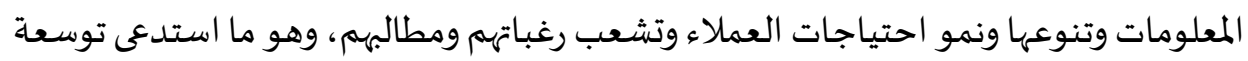

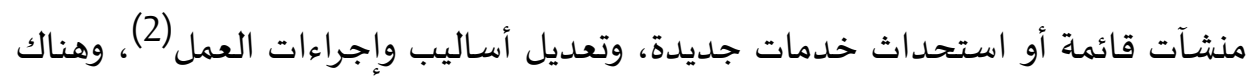
اعتقاد سائد بأن المكتبيين في السابق لم يكونوا يهتمون كثيرا بتطبيق الأسـاليب الكمية أو بتمثيل

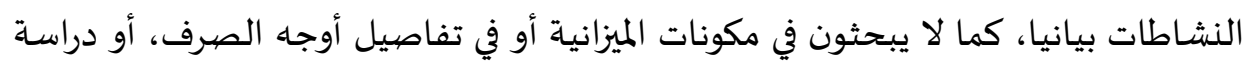

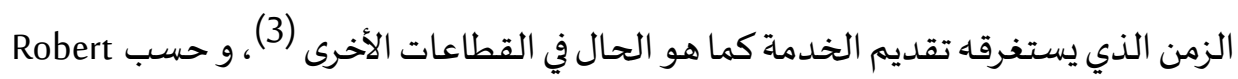

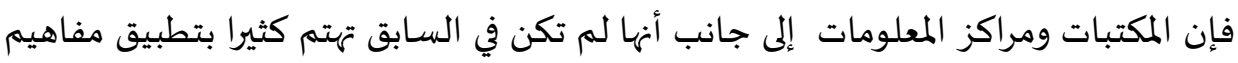
وأدوات الإدارة العلمية، فإن معظم الدراسات ومرات المعلت إنتان تلناول العمليات الداخلية دون الأخذ في الاعتبار الظروف الخارجية، كما أنها لم تدرس بشكل مفصل العاملين والعوامل المتعلقة بهم. (4)

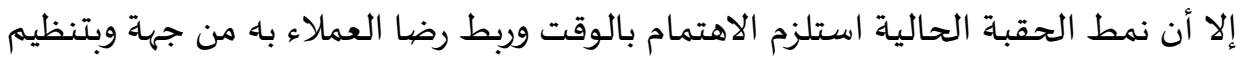

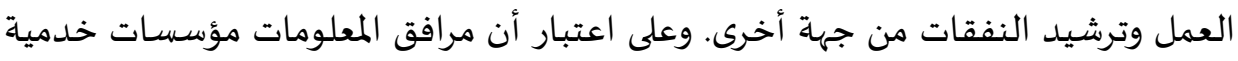

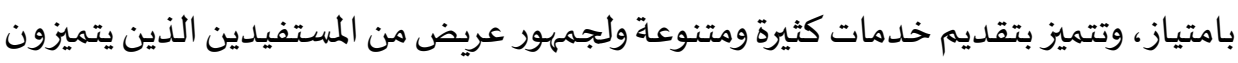

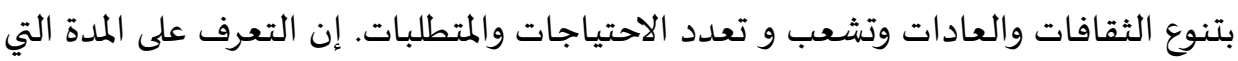

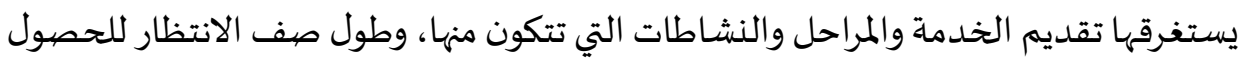

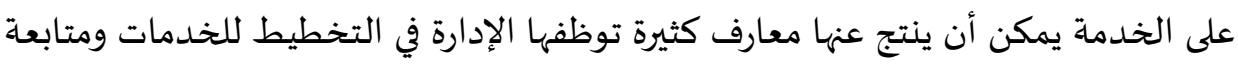


تنفيذها وتعديل وتطوير الاجراءات وفقا للمؤشرات التي ترد من التغذية الراجعة وناتج تحليل التكلفة والعائد من التكلفة.

يعد تطبيق أدوات تحليل وتصميم النظم System Analysis \& Design Tools من أهم

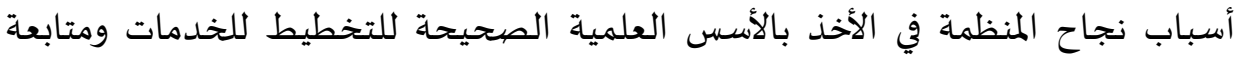

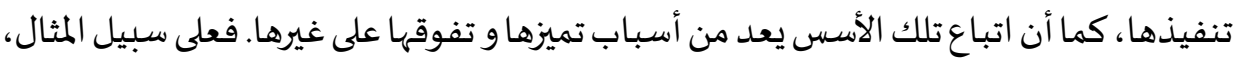

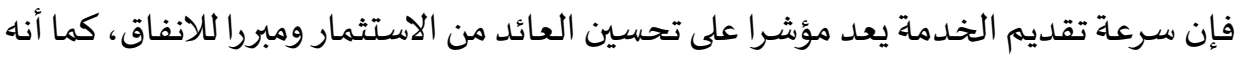

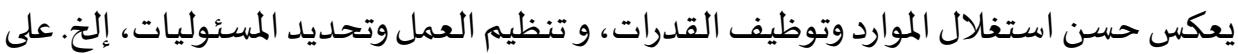

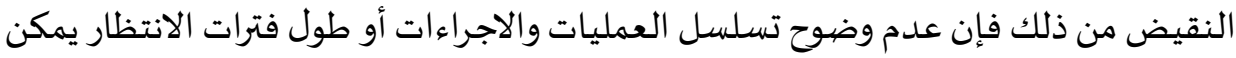

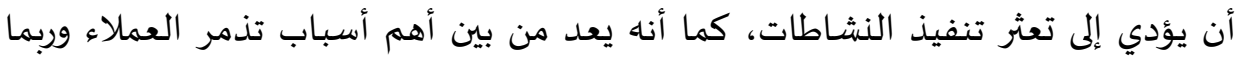

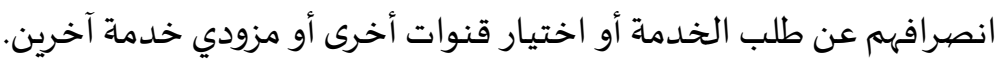

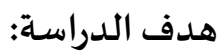

تسعى الدراسة الى تسليط الضوء على نظرية الصفوف من منطلق أنها قد تم استخدامها بنجاح في المجالات الإدارية والهندسية والعسكرية، وبالمثل فاناه يمكن توظيفها لدراسة مشككلات

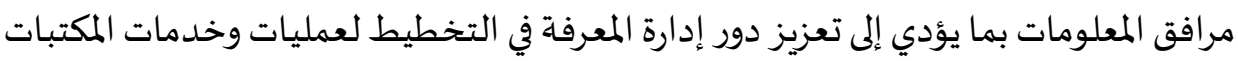

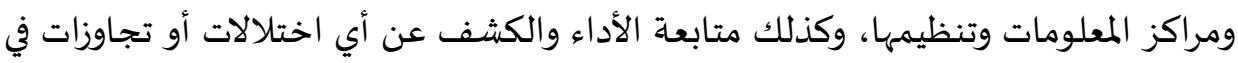

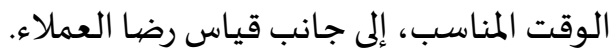

فلسفة عمل نظرية الصفوف :

تعتمد نظرية الصفوف بشكل كبير على نمذجة الظاهرة موضوع الدراسة، وتوظيف

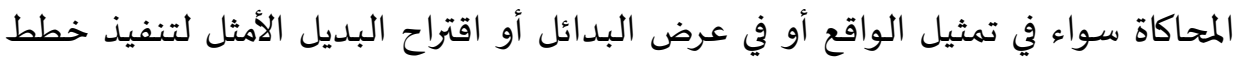

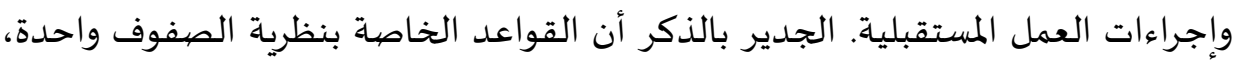

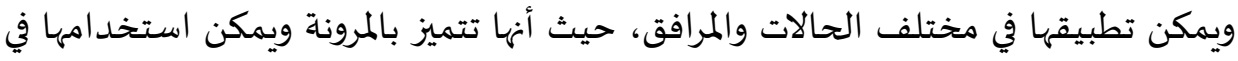

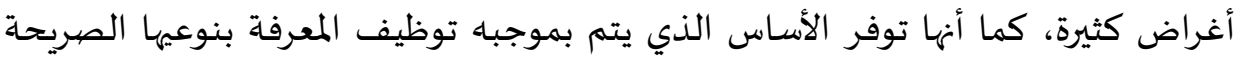

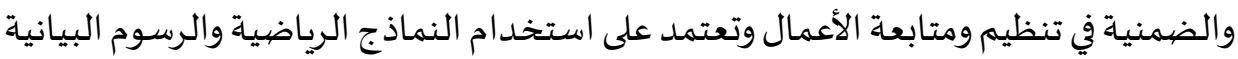

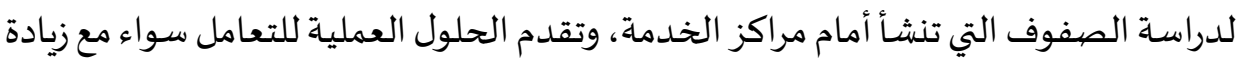


الطلب بمعدلات تفوق طاقة مراكز الخدمة، و بالعكس. وتوفر القاعدة الأسـاسية لاتخاذ القرار المناسب لأفضل إستخدام للموارد المتاحة. توظيف المعرفة في دراسة الصفوف :

المكتبة أو مركز المعلومات من المرافق الاجتماعية التي تتميز بالعمل الجماعي و تتطلب

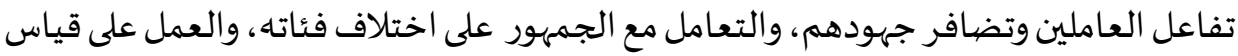

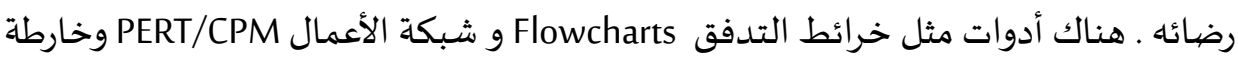
جانت Gantt Chart و نظرية الصفوف التي تستخدم في تحليل وتصميم النظمه والتي تم اقتباسها من عدة مجالات كالإدارة والهندسة والعلوم العسكرية، والتي يمكن توظيفها للتخطيط

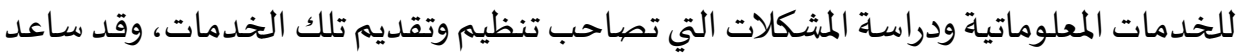
على ذلك طبيعة تخصص المكتبات والمعلومات المتداخلة مع عدد كبير من التخصصيات. من جانب آخر، يميز العمل في المكتبات ومراكز المعلومات تضخم كمية البيانات المنتجة

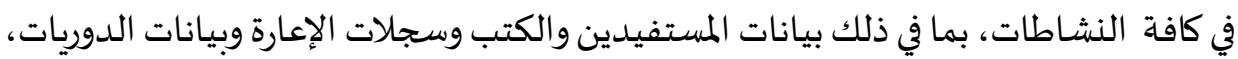
إلخ. وكان لا بد من تنظيمها وتحويلها إلى معرفة ذات قيماة يمكن استثمارها و الاستفادة منها في

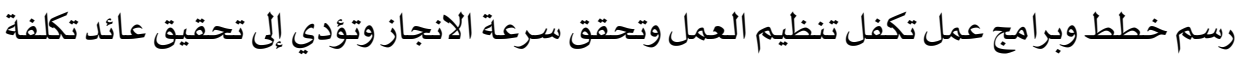
مجز للنفقات .

تهتم إدارة المعرفة بالقيام بالأنشطة التي تدخل في استكشاف المعرفة، وامتلاكها

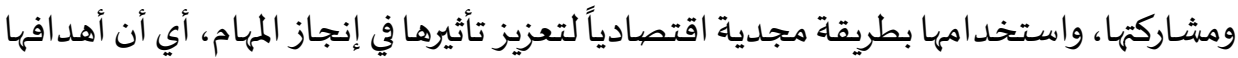
لا تقتصر على الوصول إلى المعرفة فحسب، بل تتجاوز ذلك إلى استكشافها، من خلال تحويل

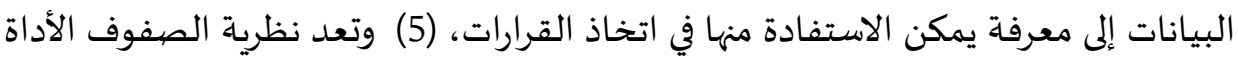
الملائمة لتحقيق ذلك.

يعد تحديد الهدف من كل نشاط تقوم به المكتبة أو مركز المعلومات من أهم الخطوات التي تسبق استخدام أي من أدوات تحليل وتصميم النظم، إلى جانب تحديد الاجراءات التهات التي

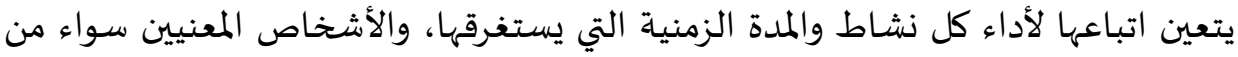
العاملين أو العملاءو التعرف على خصائصهيم وتحليل سلوكهم. إضافة إلى الأخذ في الاعتبار 
التسهيلات المتاحة والمعارف التي تم اكتسابها. ويعد اشراك العاملين والعملاء والاستفادة من

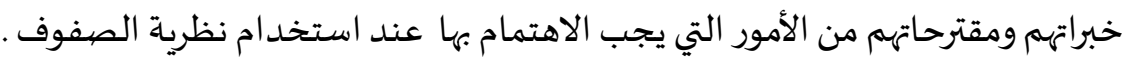
ونظرية الصفوف كأداة لجمع المعرفة يمكن توظيفها في التخطيط للخدمات المكتبية،

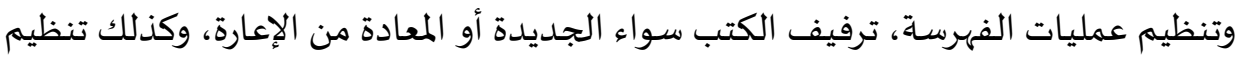

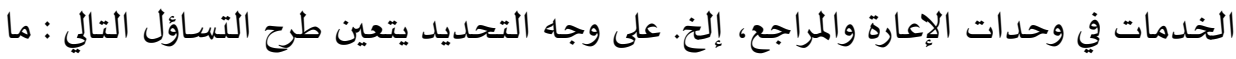

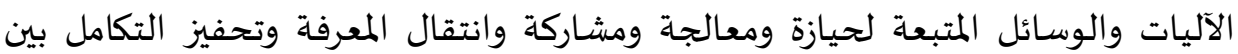
العمليات والاجرءات والوحدات ؟، وكيف يمكن توظيف تلك المعرفة في متابعة الأداء وتقييم نتائج التغذية الراجعة، والعمل على تجاوز أي هفوات أو مشكلات قد تظهر أثلات وحناء سير العمل ؟ توفر نظرية الصفوف المعرفة اللازمة التي تمكن الإدارة من إتخاذ القرارات الصحيحة

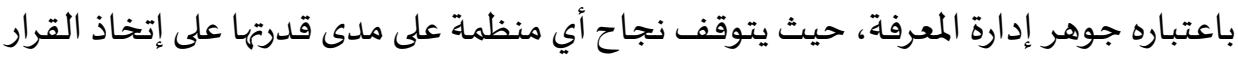

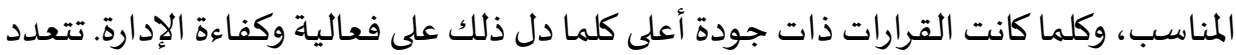

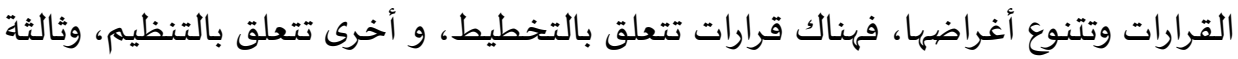

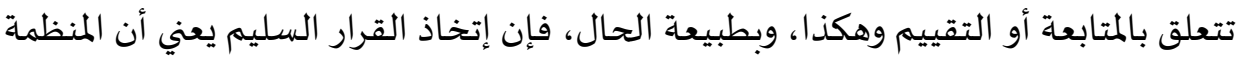

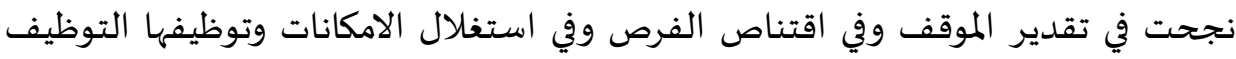
الأمثل.

تهتم نظرية الصفوف بالتفاصيل المتعلقة بالزمن الذي يستغرقه تنفيذ النشاطات، أو

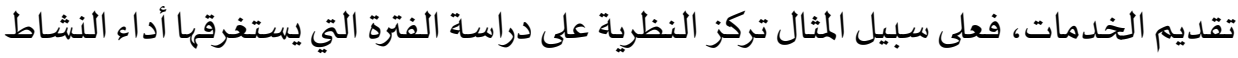

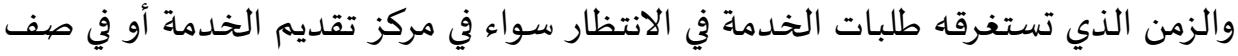

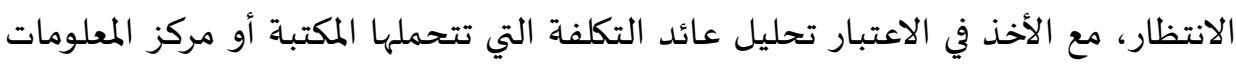

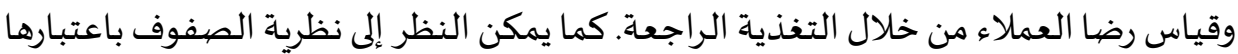

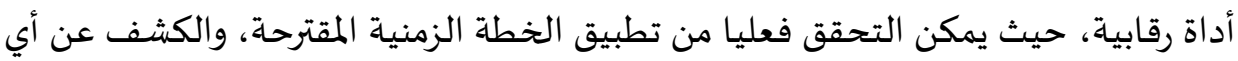
اختلالات أو هفوات في التطبيق ومعالجتها.

الواقع أن توظيف نظرية الصفوف في دراسة مراكز الخدمة وتقييم ظروف وأشكال تقديم الخدمات يسهم في تحليل العمل على أسس علمية وتصميم التوصيف الوظيفي للعاملين

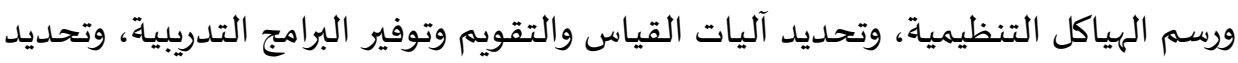


نظام المكافآت والجزاءات، وفي إعداد الميزانيات بشكل واقعي أخذا في الاعتبار الاحتياجات

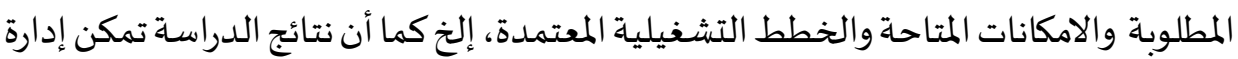
المكتبة أو مركز المعلومات من تقدير أوجه صرف بنود الميزانية، وكذلك عدد النسخ التي يتعين

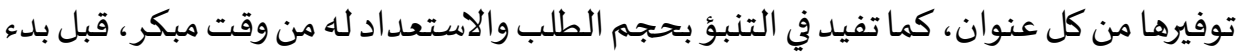
العام الدراسي مثلا، وهكذا.

من البدهي أن وقت الانتظار وطول الصف يمثلان أحد أهم مؤشرات قياس رضا العميل،

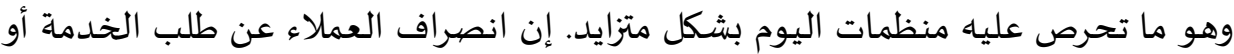

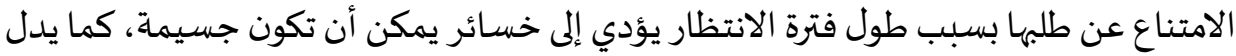

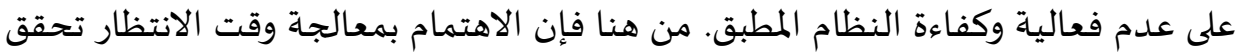
كفاءة التشغيل وجدوى الاستثمار.

على الجانب الآخر ، فإن دراسـة الصف يمكن أيضيا أن تقود إلى التعرف على كفاءة الأجهزة

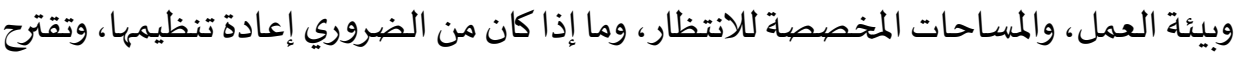
دراساة الصف تعديلات مهمة مثل : تهيئة مساحات العمل و توفير الأجواء المناسبة التي تضيف

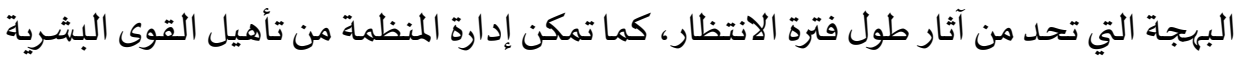

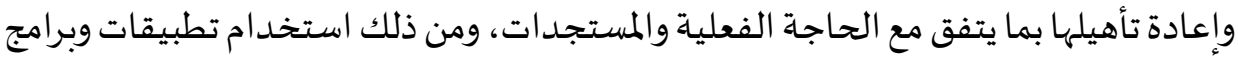
آلية متطورة لتقليص زمن تقديم الخدمة وتحسين جودة الخدمات المقدمة،وتوسيع نطاقها،

تساعد نظرية الصفوف كذلك على تحديد أسباب رضا العملاء، ومنها على سبيل المثال

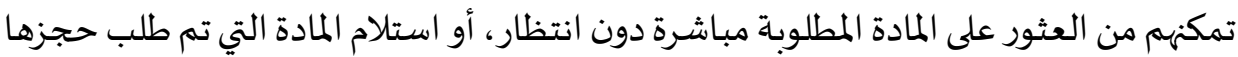
في الوقت المناسب أو الاجابة على تساؤلاتهم المرجعية في زمن قياسي، وهني، وهكذا. أسباب نشوء الصف:

يتأثر الصف بالعلاقة بين ( العرض والطلب )، ويقصد بالعرض قدرة مركز الخدمة على تقديم الخدمة، بينما يقصد بالطلب عدد الطلبات التي ترد إلى مركز الخدمة والتي يتعين تلبيتها،

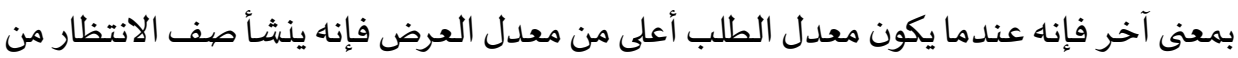


طالبي الخدمة، بينما لو كان معدل الطلب أقل من معدل العرض فإنها ينشأ صف الانتظار من

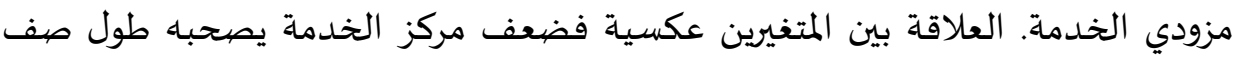

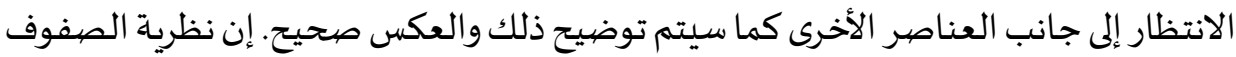
توفر معلومات عن أسباب طول الصفوف، ومن بينها على سبيل المثال : توقف الخدمة نتيجة

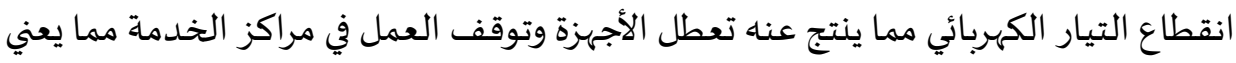
نشوء نوعين من الصفوف : صف مزودي الخدمة الذين يتوقف نشاطهم، وكذلك صفوف الخهات الانتظار التي تنشأ من طالبي الخدمة لعدم تمكنهم من الحصول على الحئ الخدمة المطلوبة، وهكذا. من أهم الجوانب التي تقع في صلب إهتمامات نظرية الصفوف كذلك : بيئة العمل، زمن تقديم الخدمة، التغذية الراجعاة، وتحليل التكلفة. فيما يلي تفصيل هذه التئيل العناصير : 1. بيئة العمل :

وتعني البيئة Environment التي يؤدى فيها العمل بما في ذلك العاملون والتسهيلات

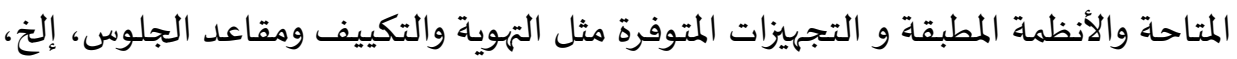

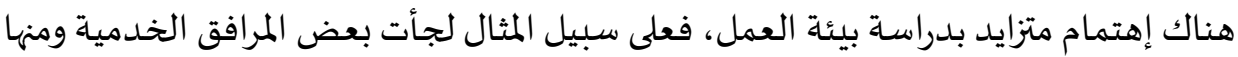

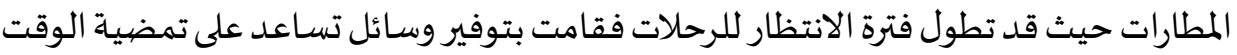

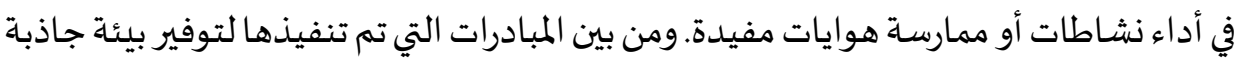

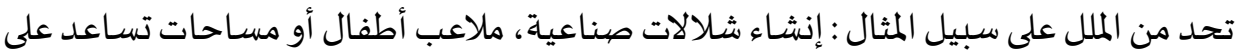
الاسترخاء والاستلقاء إلى جانب مراكز تسوق ضخمة ومتنوعة، إلخ. أما في كاونترات إنهاء

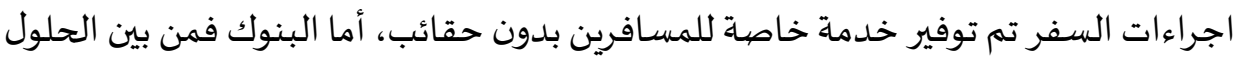

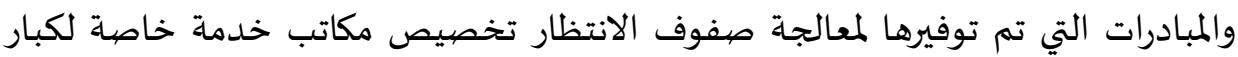

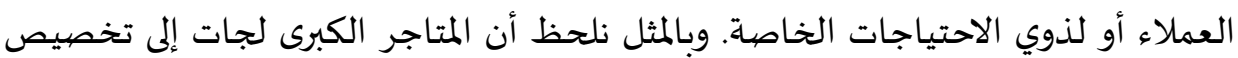

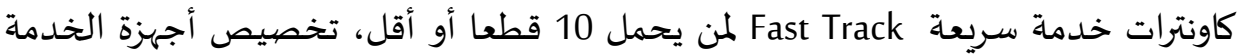

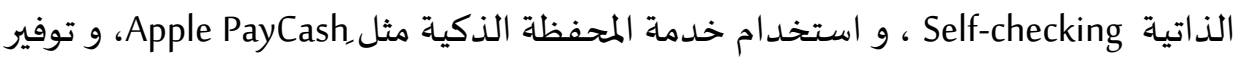

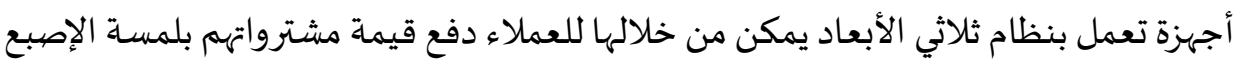

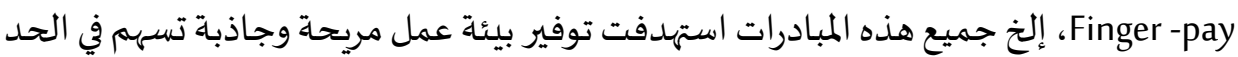


من صفوف الانتظار، وتبعا لذلك تسهم في تحقيق رضا العملاء وبطبيعة الحال في ترشيد النفقات من خلال توظيف الموارد المتاحة بأعلى كفاءة ممكنة.

\section{2. زمن تقديم الخدمة :}

تقدم نظرية الصفوف معلومات مهمة لإدارة المكتبة أو مركز المعلومات فيما يخص الكيفية التي يتم بها تقديم الخدمات والزمن المخطط له لتنفيذ كل خدماة، فعلى سبيل المثلال:

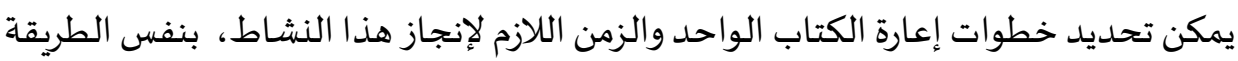

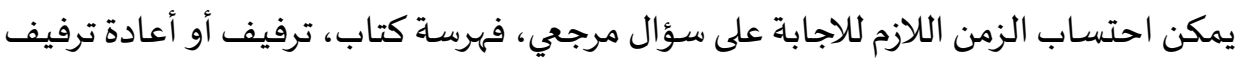

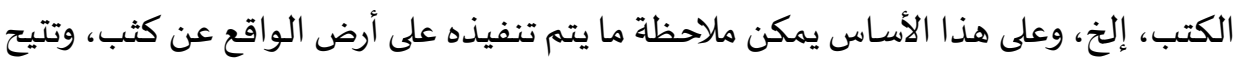

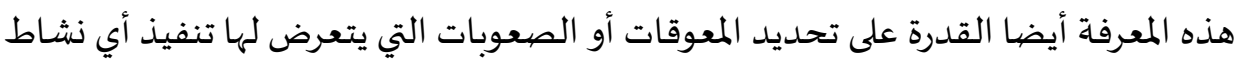

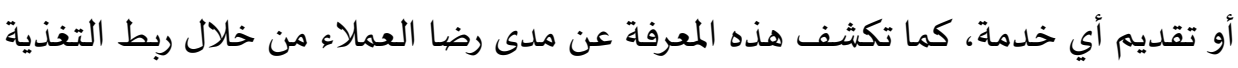

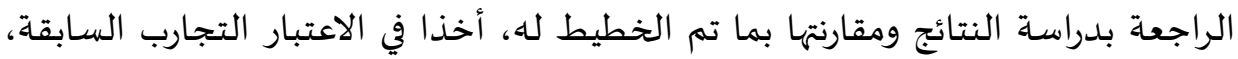

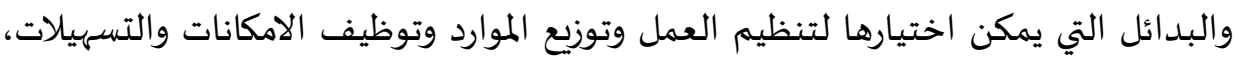

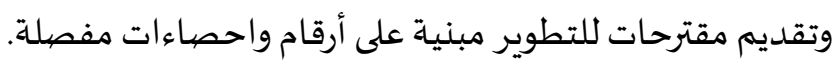

وللتعرف على العلاقة بين رضا العميل وزمن الانتظار يوضح الجدول رقم ( 1 ) مثالا للعلاقة العكسية بينهما، حيث أنه كلما قل زمن الانتظار كلما أدى ذلك إلى تحقيق درجاة أعلى العانى من رضا العملاء، وهكذا.

جدول رقم (1) العلاقة بين رضا العميل وزمن الإنتظار

\begin{tabular}{|c|c|}
\hline زمن الإنتظار(دقيقة) & 15 \\
\hline 2 & 11 \\
\hline 4 & 6 \\
\hline 7 & 3 \\
\hline 10 & \\
\hline
\end{tabular}




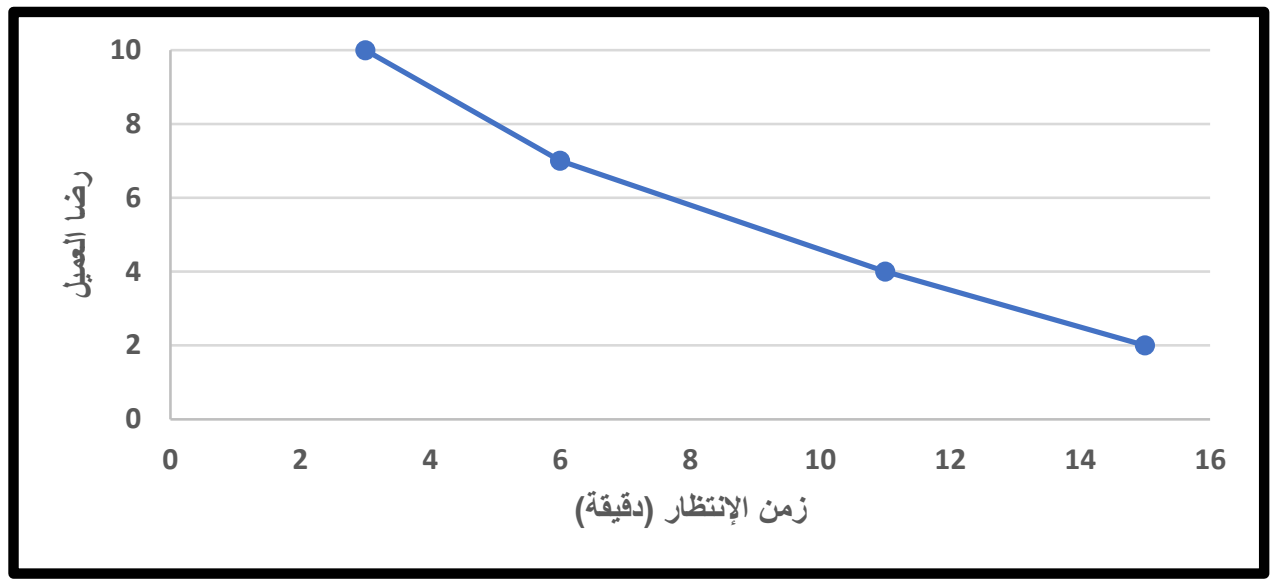

شكل رقم (1) العلاقة بين رضيا العميل وزمن الإنتظار

3. - ت تحليل التكلفة :

تعتبر نظرية الصفوف أداة هامة لتحليل التكلفة، حيث أن احتساب تكلفة الانتظار توفر

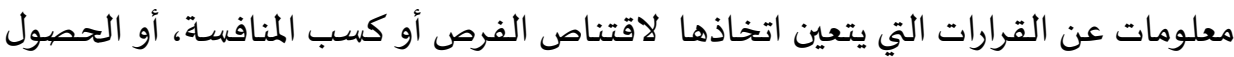

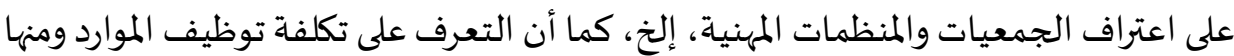

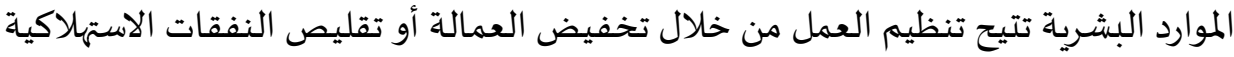

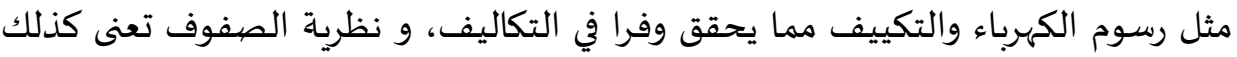

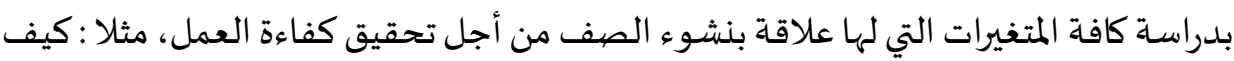
يتشكل الصف، مم يتكون، وكيف يتحرك الصف، ونمط تقديم الخدمة، مما يتيح مراعاة

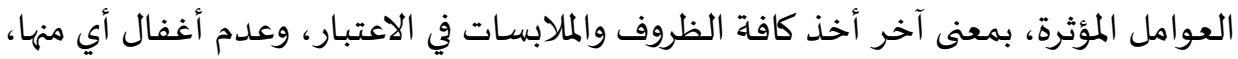

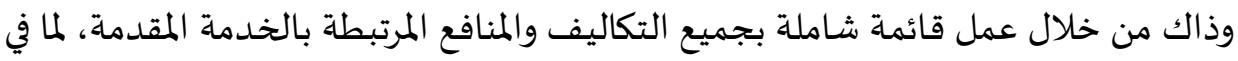
ذلك التكاليف المباشرة وغير المباشرة والتكاليف غير الملموسة وتكاليف الفرص وتكاليف

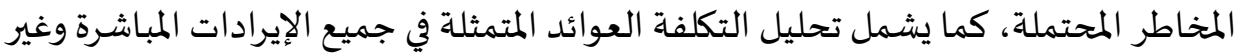

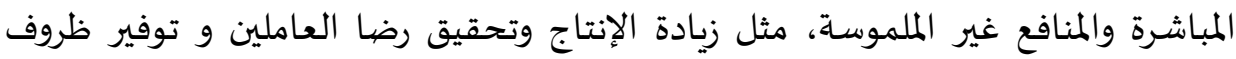
وتنظيمات عمل محفزة، ومن ثم تتم المقارنة الكمية بين نتائج تحليل التكلفة والعائد الإجمالي

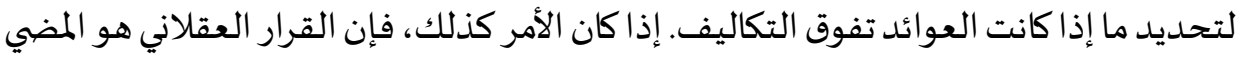


قدما في تنفيذ الخطط وإجراءات العمل المقررة سلفا. (6)، كما يرتبط تحليل عائد التكلفة بشكل

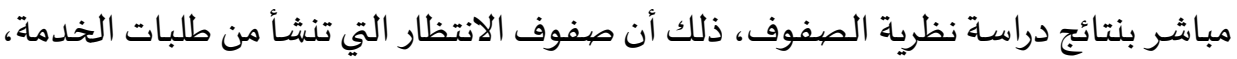

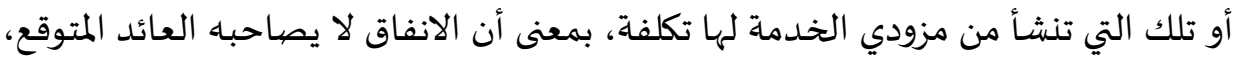

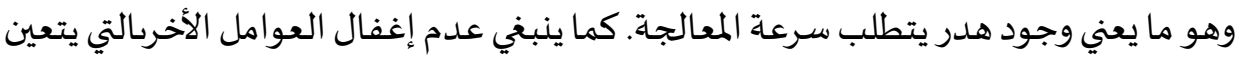

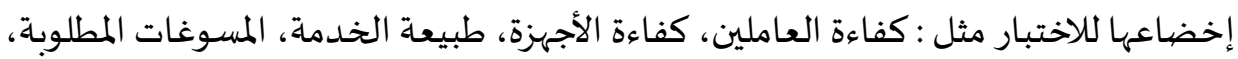

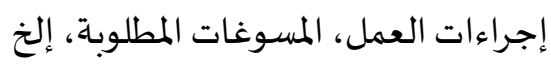

وكمثال لاحتساب التكلفة الكلية للخدمة يتم تطبيق المعادلة التالية : التكلفة الكلية = تكلفة تقديم الخدمة + تكلفة الانتظار

يوضح الجدول رقم (2) مثالا للعلاقة الطردية بين تكلفة الخدمة ومستوى الجودة جدول رقم (2) العلاقة بين مستوى الخدمة المقدمة و تكلفة تقديمها

\begin{tabular}{|c|c|}
\hline مستوى الجودة & تكلفة الخدمة (الف ريال) \\
\hline 10 & 100 \\
\hline 7 & 80 \\
\hline 4 & 50 \\
\hline 3 & 30 \\
\hline
\end{tabular}

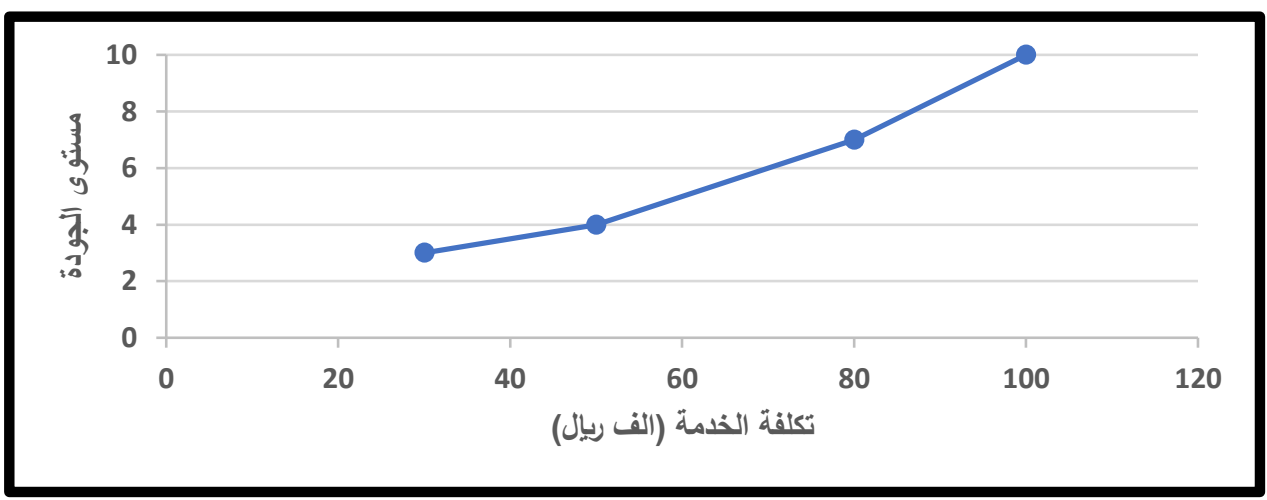

شكل رقم (2) العلاقة بين مستوى الخدمة المقدمة و تكلفة تقديمها 


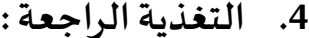

يشكل قياس رضا العملاء من أهم مقومات تطبيق نظرية الصفوف، فمن خلال التغذية الراجعة التي يتم الحصول عليها، والتي توفر المعرفة بنوعيها الصريحة والضمنية، بالإضافة إلى تحليل العمل تتوفر المعارف الضرورية التي تمكن الإدارة من إتخاذ القرارات اللازمة للتعامل مع النظام ومكوناته وموارده وتحليل البيئة التي يتم فيها تنفيذ العمليات موضوع تطبيق نظرية الصفوف. هناك عدة أساليب لقياس رضا العملاء. توضح الأشكال ( 3 ، 4، 5 5، 6 ) بعض آليات قياس رض العملاء، مع ملاحظة أنها تكون متاحة قبل و أثناء أو بعد الانتهاء من

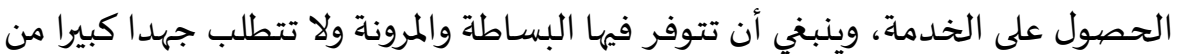

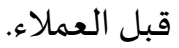

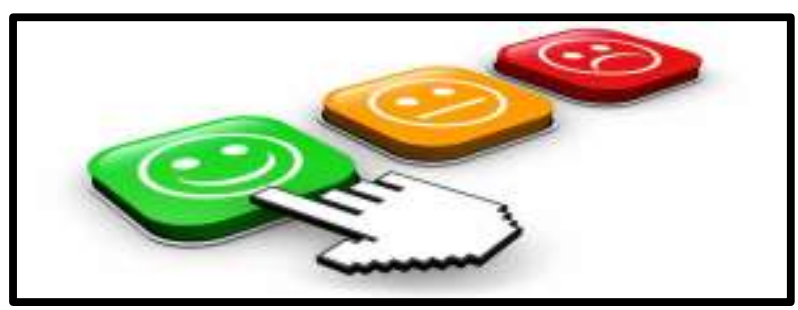

$$
\text { شكل رقم ( } 3 \text { ) التغذية الراجعة ( لوحة إلكترونية ) }
$$

/https://ontopvisibility.com/important-reasons-customer-feedback : المصدر

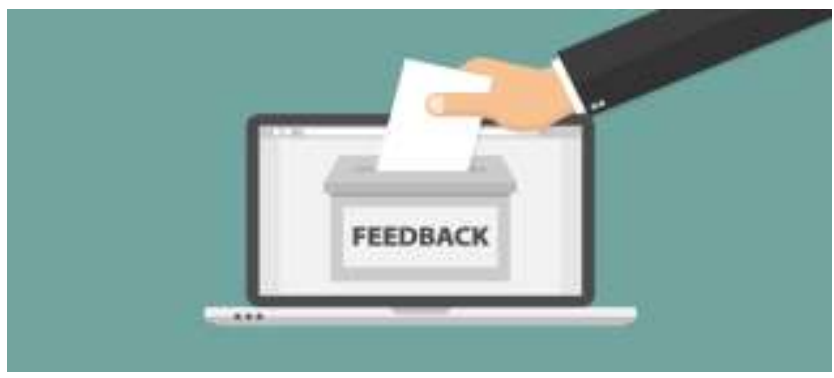

$$
\text { شكل رقم ( } 4 \text { ) التغذية الراجعة ( صندوق اقتراحات ) }
$$

https://wpforms.com/how-to-add-a-customer-feedback-form-to-your- : المصدر $\underline{\text { wwordpress-site }}$ 


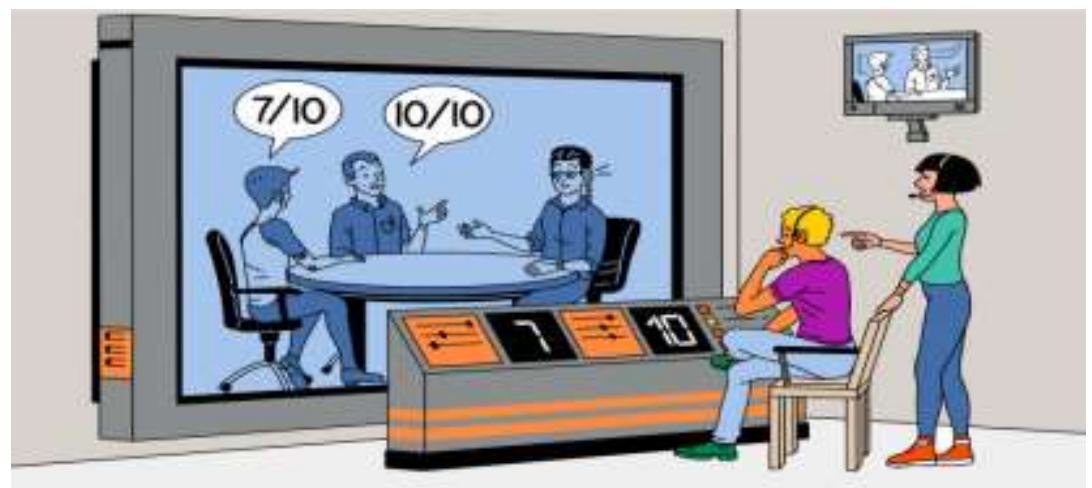

شكل رقم ( 5 ) التغذية الراجعة ( غرفة محادثة)

$$
\text { المصددر : }
$$

https://images.ctfassets.net/1893v89mix1e/3edEk5wYaxWJPxkFWDySQD/17d76 11f8e8c96815b89813eb6f003da/Customer_feedback_16x9_hero.jpg?w=750\&h $=422 \& \mathrm{fl}=$ progressive $\& \mathrm{q}=80 \& \mathrm{fm}=$ jpg

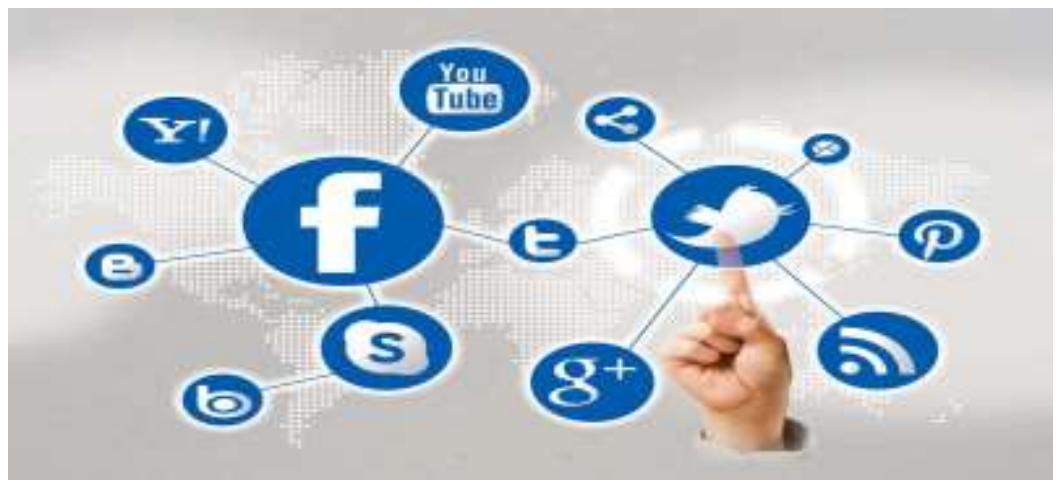

$$
\text { شكل رقم ( 6) التغذية الراجعة }
$$

https://helpjuice.com/blog/5-ways-to-ask-for-customer-feedback: المصصدر

يرتبط نشاط نظرية الصفوف بشكل مباشر بنتائج التغذية الراجعة، ذلك أنها تعني

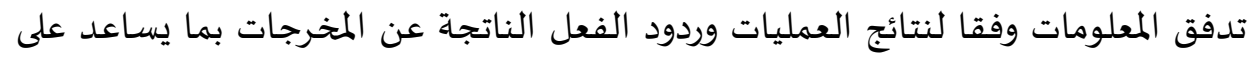
التحقق من أداء العمل حسب الأهداف المحددة والكشف عن أوجه الخلل في الوقت المناسب. 
يمكن أن تكون التغذية الراجعة وقتية بعد الحصول على الخدمة مباشرة أو أثناء عملية

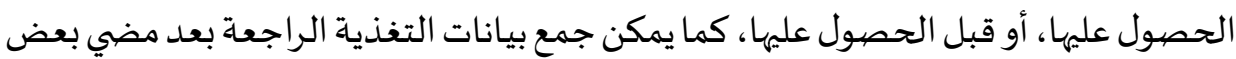

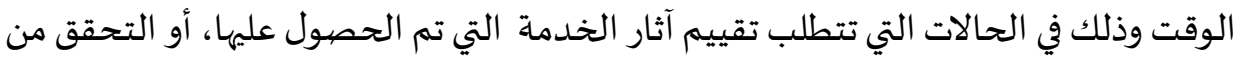

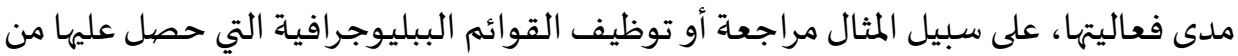

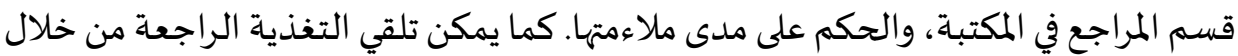

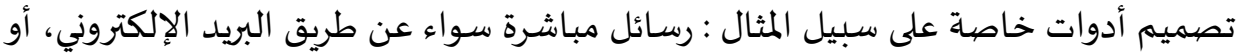

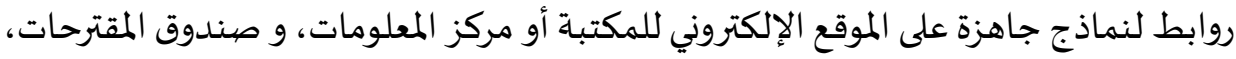

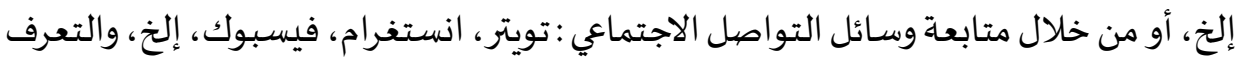

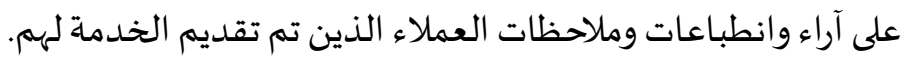
يمكن أن تكون التغذية الراجعة عامة و يطلع عليها الجميع مثل: التعليقات في وسائل

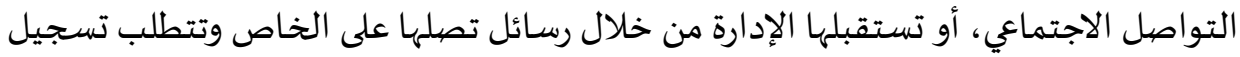

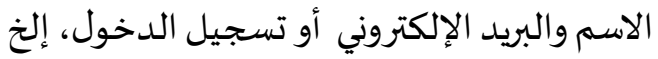
تأخذ التغذية الراجعة أحد شكلين كما يلي :

\section{تغذية راجعة سلبية :}

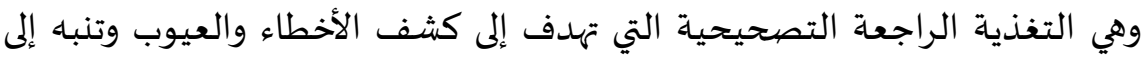

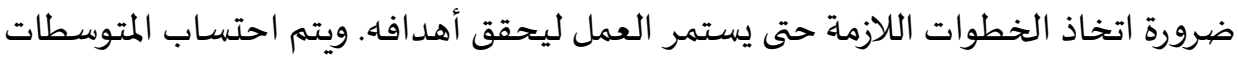

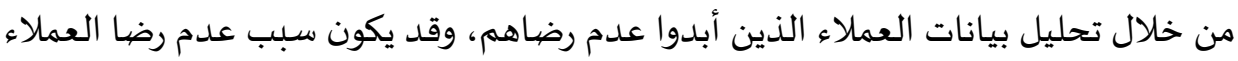

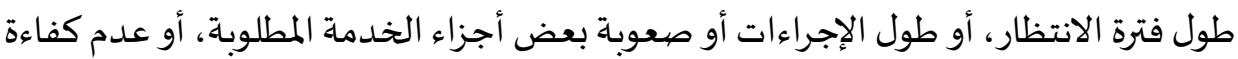

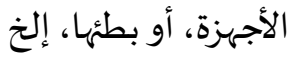

\section{تغذية راجعة إيجابية :}

وهي التغذية الراجعة التي تدل على أن العمل يسير وفقا لما هو مخطط له أو أفضل،

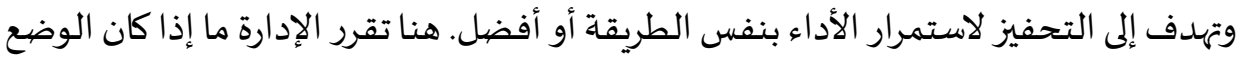

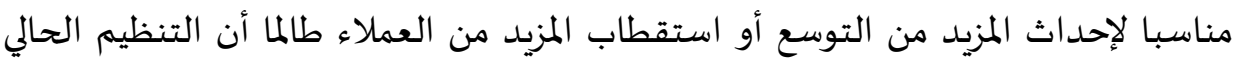
والخدمات المقدمة تحظى برضيا العملاء، وهكذا... 
طريقة عمل نظرية الصفوف :

توظف نظرية الصفوف النماذج الرياضية - كما سبق إيضاحه - للتعامل مع زيادة

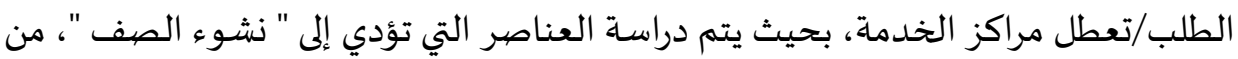

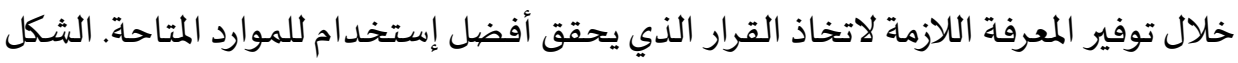
رقم ( 7 ) يوضح فكرة نظرية الصفوف، حيث يتضح أهها تتكون من 4 مراحل كما يلي :

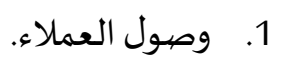

2. انتظامهم في صف أو جلوسهمى وفق موعد الوصهول أو حسب الأرقام التي حصلوا عليها

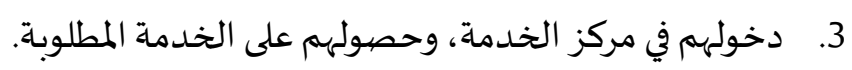

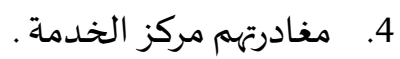

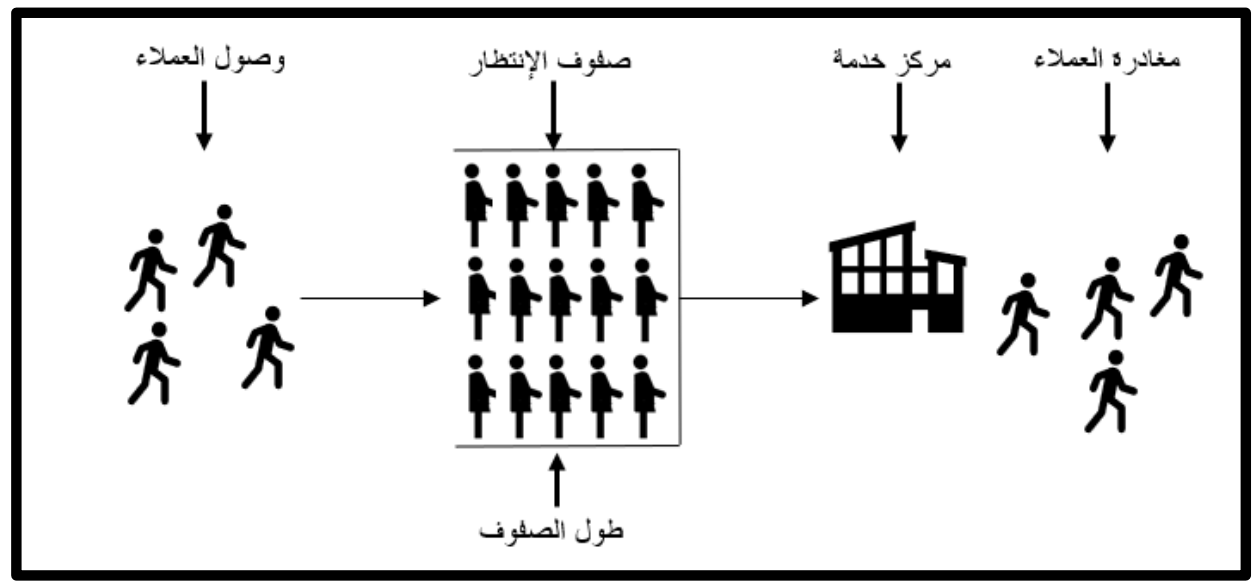

شكل رقم (7 ) مراحل دراسة صفوف الانتظار

فيما يلي تفصيل هذه المراحل :

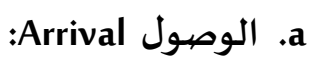

يتعين التعرف على نمط الوصول ( جماعات، أفراد)، توقيت الوصول، عدد

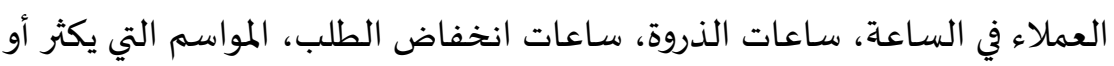

يقل فهيا الطلب على الخدمة، طبيعة طالبي الخدمة وثقافتهم نحو الالتزام بالصف وإعطاء أولوية لمن يسبقهم أو لذوي الاحتياجات الخاصة أو المرأة أو كبار السن، إلخ. 
bue Q الصف: bue

من حيث حركة الصف، على سبيل المثال هل من الأفضل تنظيم طالبي الخدمة في صف واحد ومن ثم يتم انتقالهم لمركز الخدمة المتاح حسب الأرقام، أو أن يتم تنظيم طالبي الخدمة في صفوف متعددة، بنظام صف أمام كل مركز خدمة، وهكذا . مع ملاحظة وجود نوعين من صفوف الانتظار : صف في مركز الخدمة (مبنى المكتبة

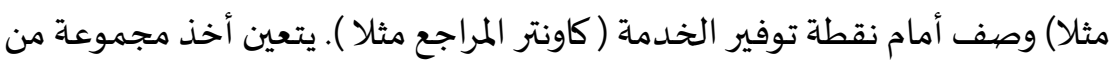
العناصر في الاعتبار عند دراسـة الصف منها :

- هل يتم تنظيم الخدمة تلقائيا في مراكز خدمة محددة سلفا للفئات الخاصة؟

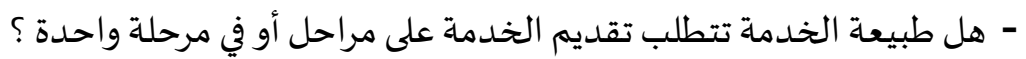
- ما أثر الشعور النفسي في حالة الصف الواحد أو الصفوف المتعددة، بمعنى هل تلماحل يلزم الانتظار في صف واحد طويل ومن ثم الانتقال إلى مراكز الخدماة، أو أن أن يخصص لكل مركز خدمة صف انتظار مستقل، بحيث يبدو الصف في الحالة

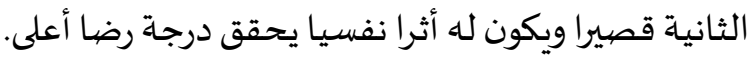
- ما المساحة المتاحة للانتظار ؟

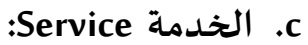

هي المرحلة التي يتم فيها تقديم الخدمة فعليا، ويتعين التعرف على مجموعة من

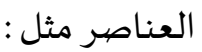

- هل تتوفر نقاط الخدمة السريعة ؟ مثل : عملاء من ذوي الاحتياجات الخاصة،،

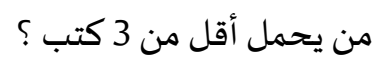

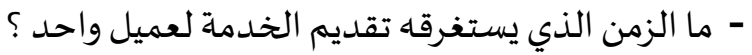
- ما فعالية وجاهزية مقدم الخدمة لتقديم الخدماة، بما في ذلك كفاءة العناصري

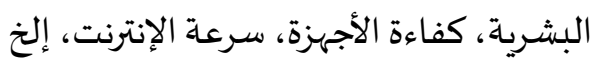

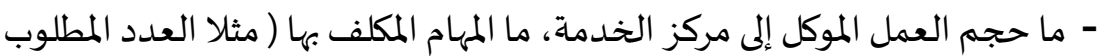

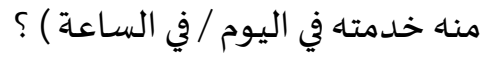


- ما قواعد الخدمة المعتمدة هل من يأت أولا تقدم له الخدمة أولا أو أن هناك نمطا

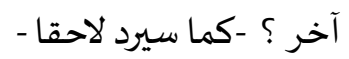

d Departure المغادرة d

هي المرحلة التي يغادر فيه طالبوا الخدمة الصف، ومن ثم مركز الخدمة، ويتعين التعرف على مجموعة من العناصر مثل :

- هل تتوفر مساحات خاصة للمغادرين غير تلك المخصصية للقادمين لتحقيق انسيابية الحركة ؟ هل توفر مساحات

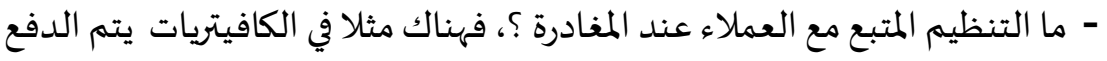

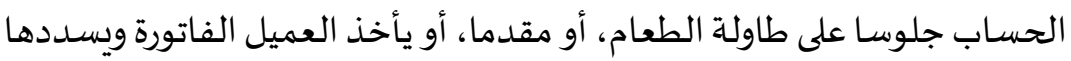
بنفسه عند الكاشير.

- هل حصل طالبوا الخدمة فعليا على الخدمة بشكل كامل ؟ أو جزئيا ؟ ؟ - هل تمت المغادرة دون الحصول على الخدمة مما يتطلب معاودة الطلب لاستكمال شروط أو مسوغات معينة، إلخ. نمذجة ومحاكاة الصفوف :

تعد النمذجة Modeling والمحاكاة Simulation مهمة جدا في عمل تحليل وتصميم النظم، بل من وجهة نظر هذه الدراسة تعتبران حجر أساس التحليل، ومن أهم أسباب نجاح دراسـة أي نظام للصفوف، كما أهما من أهم الوسائل التي تساعد على فهم الأنظمة المعقدة المها

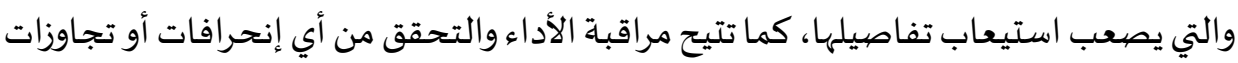

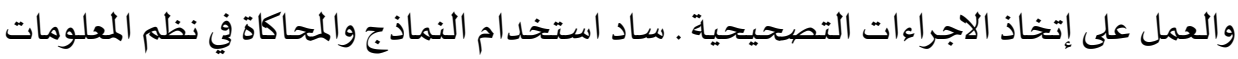

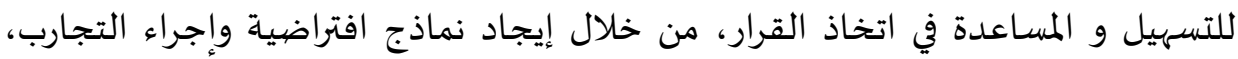

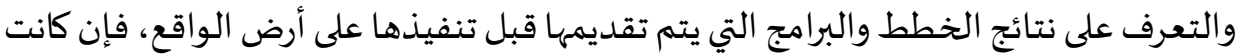
وفق المتوقع والمرغوب طبقت على الأنظمة الحقيقية وإن لم تكن كذلك أجريت عليها التعديلات 
تعني النمذجة التمثيل المنطقي أو الرياضي لصف الانتظار بطريقة تحاكي التنظيم

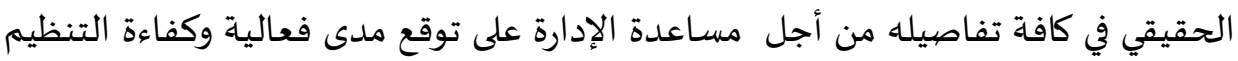

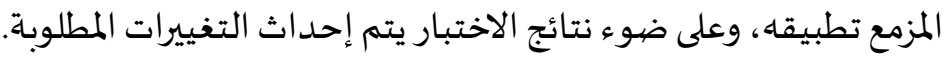

أما المحاكاة فتعني تشغيل النموذج الذي يتم تصميمه أخذا في الاعتبار عناصر مهمة

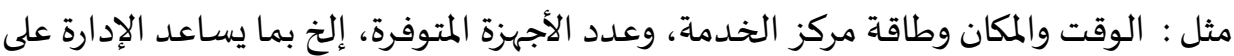

تحليل الأداء سلفا. (7) تولت

ينتج عن توظيف النمذجة والمحاكاة عدد من المزايا كما يلي :

- تشخيص طريقة العمل وانسيابية الأداء افتراضيا، و تسلسل الاجراءات و عدد

نقاط الخدمة التي يتعين توفيرها

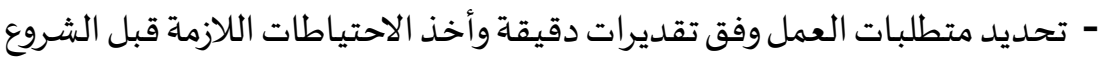

في تنفيذ الخطة على أرض الواقع.

- التعرف على المعوقات أو الصعوبات التي يمكن أن يواجهها النظام. لواتع.

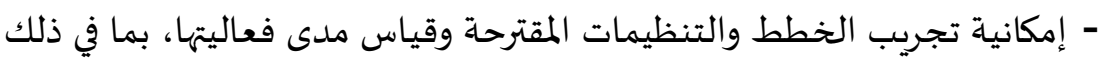

$$
\text { قياس الوقت اللازم لتقديم الخدمة. }
$$

- التعرف على العوامل التي توثر على رضا العملاء.

- التعرف على كيفية التشغيل الفعال لمراكز الخدمة بأقصى طاقة ممكنة.

$$
\text { - متى ينشأ الصف ؟ الصفئ }
$$

يمكن تمثيل مشكلة الصف الذي يتكون من مجموعة من العملاء الذين يقفون في صف

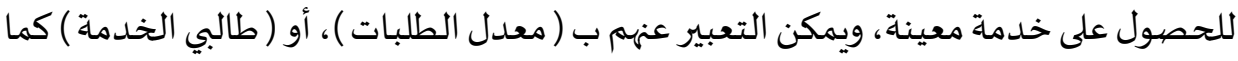

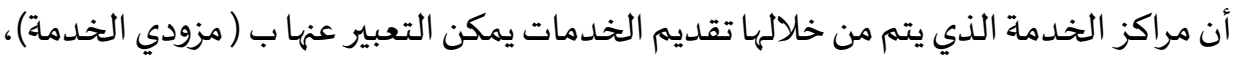

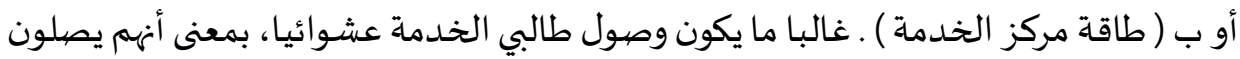

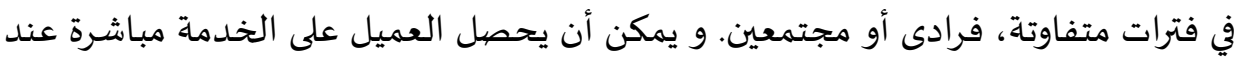

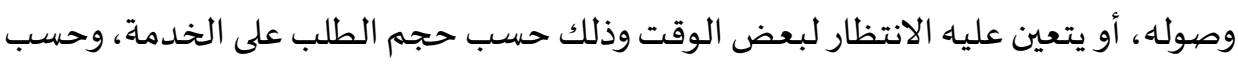

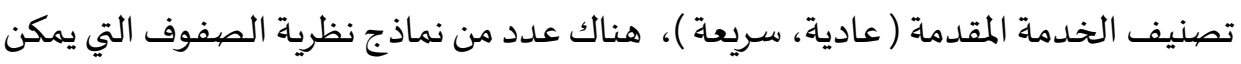




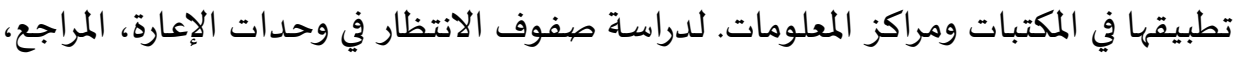

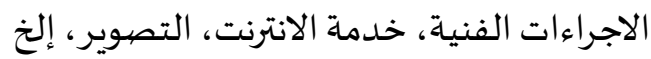

الجدير بالملاحظة أن صفوف الانتظار لاتعني فقط تلك الصفوف التي تنشأ أمام مراكز

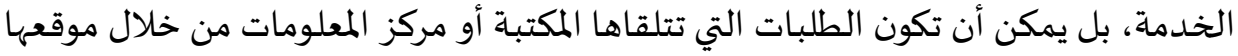

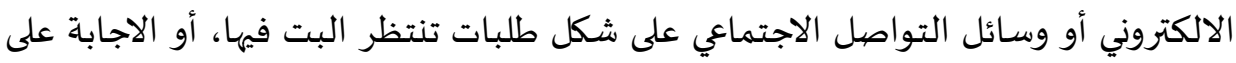

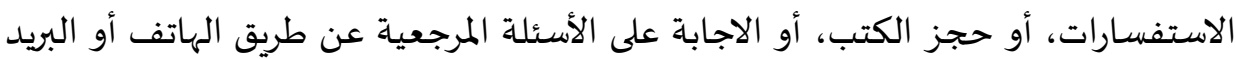

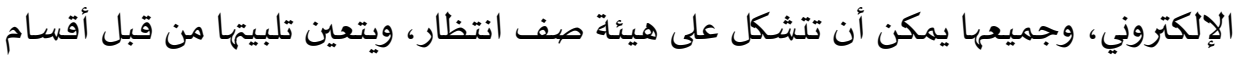

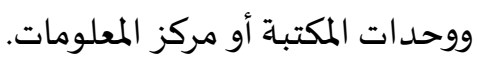

يمكن النظر إلى العلاقة بين معدل الطلبات و طاقة مركز الخدمة على إنها ( علاقة عكسية ) كما يلي : nمن النغر

\section{طلبات خدمة أكثر من طاقة مركز الخدمة :}

بمعنى أن طالبي الخدمة يفوق عددهم عن طاقة مركز الخدمة، و ينتج في هذه الحالة

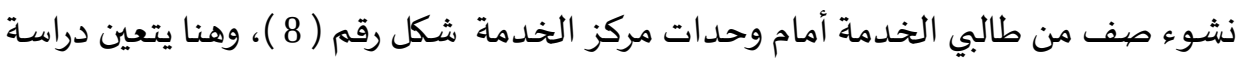

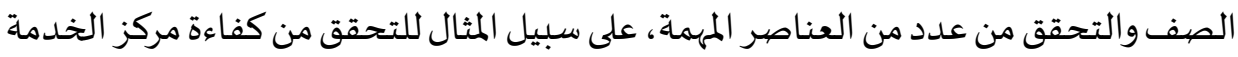
يتعين معرفة ما يلي :

$$
\begin{aligned}
& \text { - عدد العاملين } \\
& \text { - الاجراءات المطلوبة - } \\
& \text { - الوقت المستغرق لإنجاز المهمة } \\
& \text { - سلوك العاملين والعملاء } \\
& \text { - المسوغات المطلوبة سلماتية }
\end{aligned}
$$

- عمليات ما قبل طلب الخدمة، مثلا رفع المسوغات إلكترونيا، اجراء اختبارات

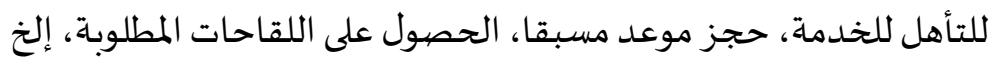




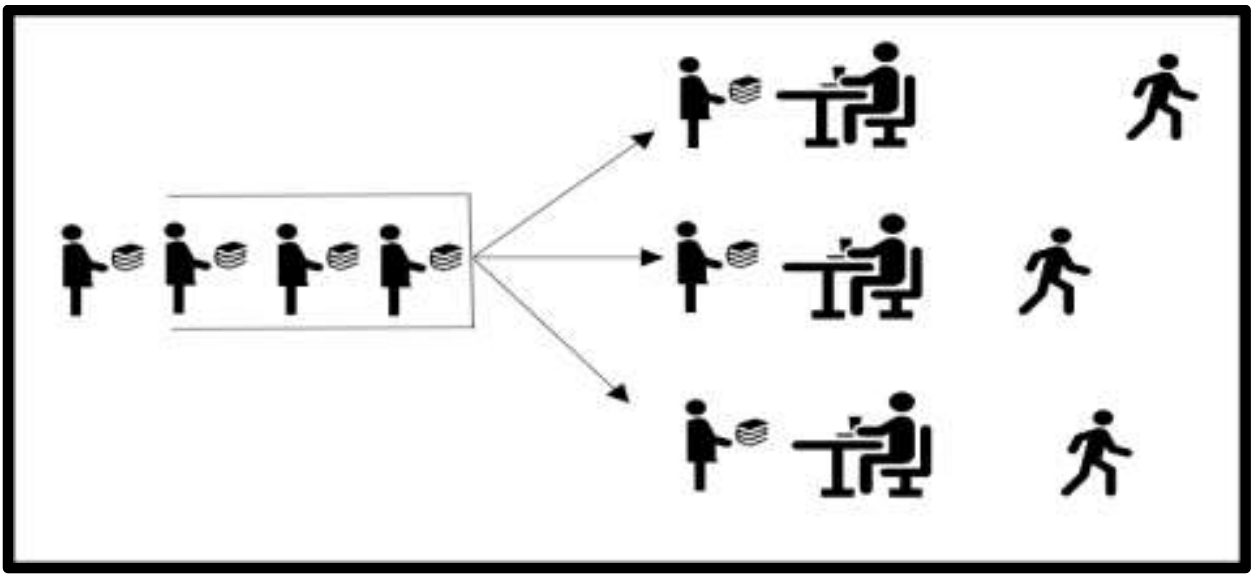

شكل رقم ( 8 ) تمثيل حالة طلبات أكثر من طاقة مركز الخدمة

• طلبات خدمة أقل من طاقة مركز الخدمة:

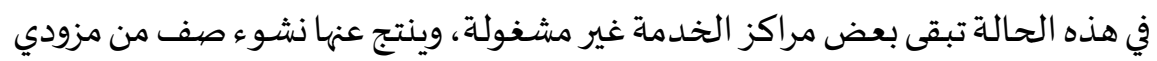

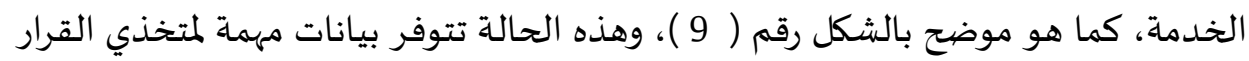

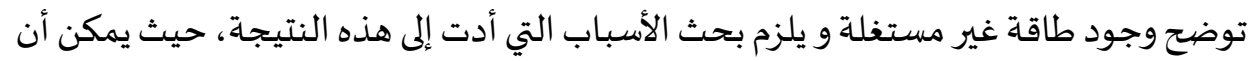

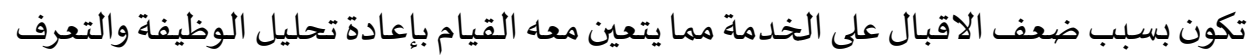
على سبب احجام العملاء عن طلب الخدمة ( مثلا :وجود منافسين، اكتفاء العملاء ذاتيا، سوء أخداء

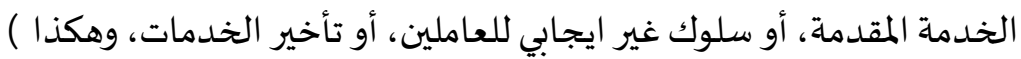

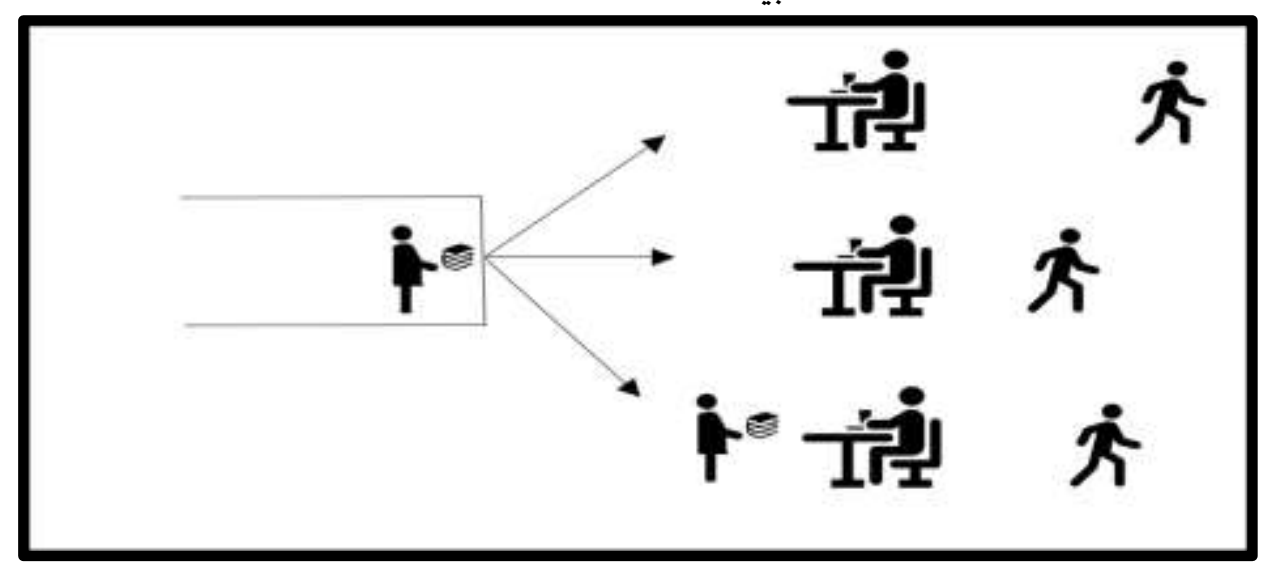

شكل رقم ( 9 ) تمثيل حالة طلبات أقل من طاقة مركز الخدمة 
تنشأ عن طول فترة الانتظار للحصول على الخدمة المطلوبة نتائج تختلف من حالة إلى

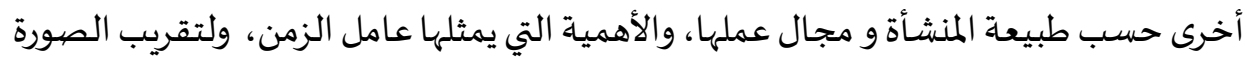

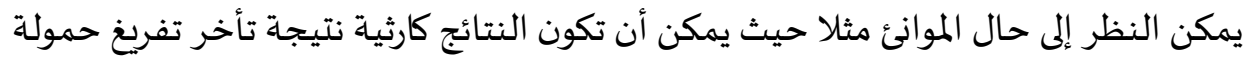

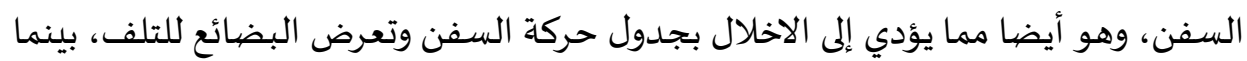

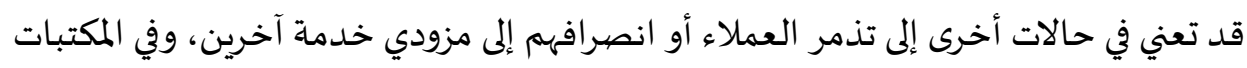

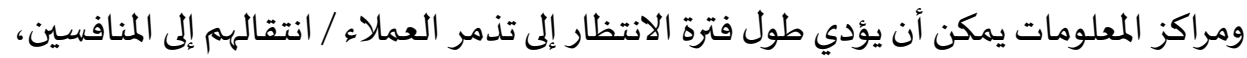

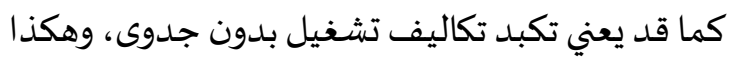

هناك مجموعة من العوامل المؤثرة التي يمكن أن تسهم في نشوء الصف وفي طوله أو قصره، يمكن تقسيمها إلى (5) انواع كما يلي :

أولا : ظروف مر اكز الخدمة، وتتمثل في :

- طاقة مركز الخدمة والتجهيزات المتاحة. - - نقص أو غياب العاملين أو عطل بعض الأجهزة. - القدرة الاستعابية لغرف وصالات الانتظار.

ثانيا : معدل وصهول الطلبات، وتتمثل في :

$$
\begin{aligned}
& \text { - وصهول طلبات الخدمة فرادى. } \\
& \text { - وصولهم على شكل مجموعات. } \\
& \text { ثالثا : السلوك ويتمثل في : }
\end{aligned}
$$

- - ملب العميل لخدمة من مركز خدمة خطا.

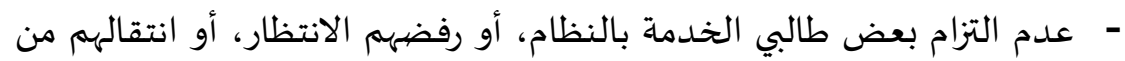

$$
\text { صف لآخر. }
$$

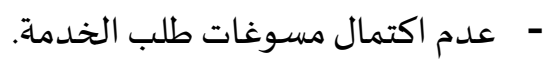
- ت تعدد الطلبات في الزيارة الواحدة. 


\section{رابعا : قواعد الخدمة، وتتمثل في (3) أشكال كما يلي :}

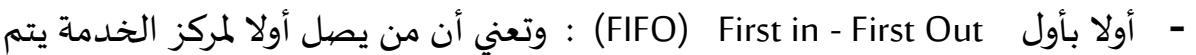

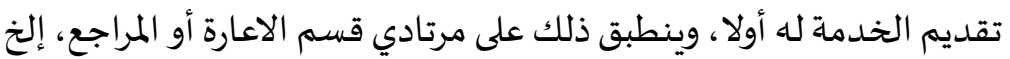

- بحسب الأولوية Priority) : وتعني أن لا يتم الأخذ بمعيار أولوية الوصيول لتقديم

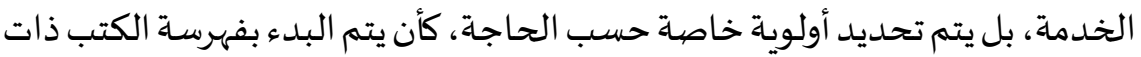

العلاقة بالمقررات الدراسية أولا في حال قرب موعد بد بدء العام العام الدراسي.

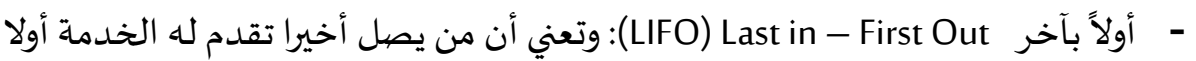

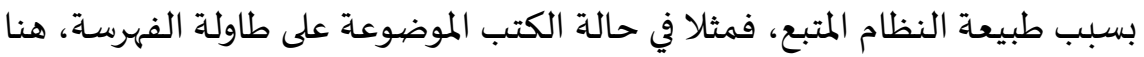

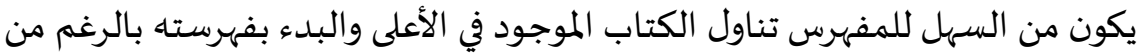

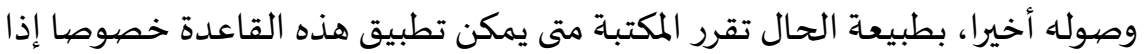
كانت المطلوب فهرسة جميع الكتب التي وردت للقسم دون أن تكون هناك أولوية معينة. الشكل رقم ( 10 ) يوضح حالة الكتب التي يتم فيها تطبيق هذه القاعدة، حيث يمكن تخيل ورود الكتب في فترات متفاوتة، وتم وضعها رأسيا فوق بعضها البعض. هنا يكون من فن السهل تناول الكتاب الذي وصل أخيرا لتتم فهرسته أولا.

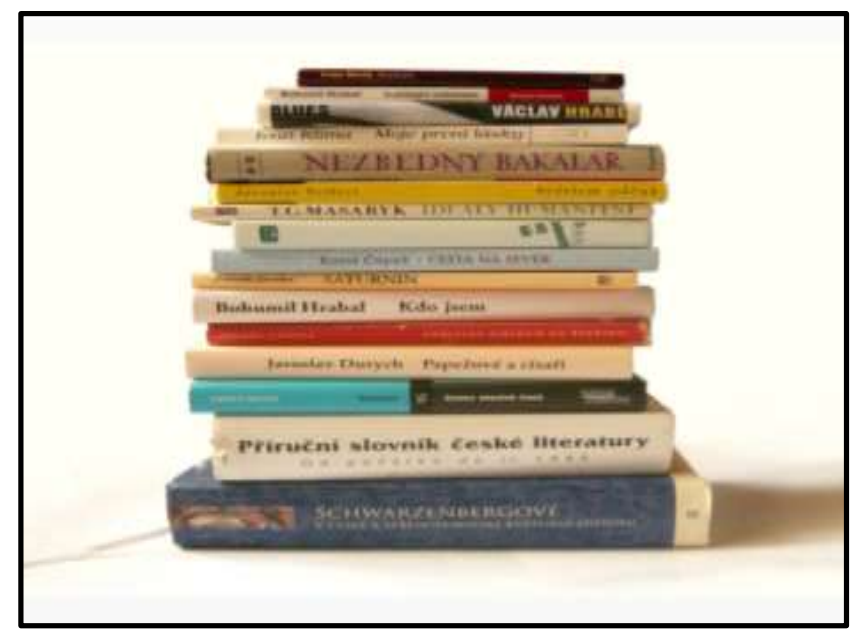

شكل رقم (10) صف كتب بانتظار خدمة الفهرسة بنظام أولا بآخر

Download 2100K+Free Clipart on Jooinn : المصدر 
خامسا : ظروف تقديم الخدمة :

ويقصيد بها متطلبات تقديم الخدما في فترات زمنية معينة كالمواسم التي يزيد فيها الطلب

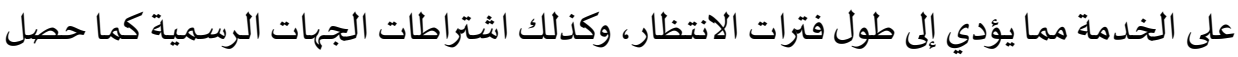
خلال فترة جائحة كورونا، حيث تعين الابقاء على مسافة محددة بين كل عميل وآخر، وكذلك الك

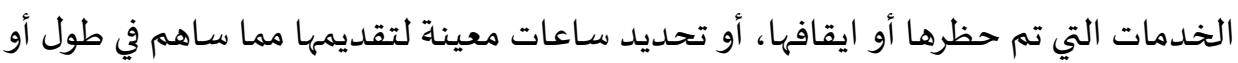

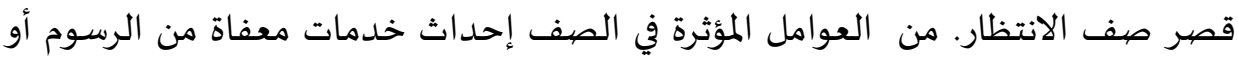

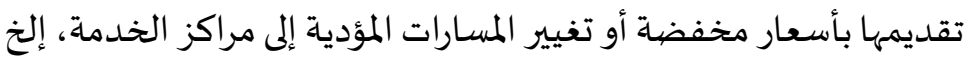

أشكال الصفوف : تأخذ صفوف (طالبي الخدمة)، وكذلك ( مزودي الخدمة) عدة أشكال كما : يلي : 1. حالة صف واحد، ومركزخدمة واحد : يمكن ملاحظة مثل هذه الحالة في الوحدات الصغيرة التي يتم فيها الاكتفاء بمركز واحد ينشأ أمامه صف واحد من طالبي الخدماة، كما في شكل رقم ( 11 ).

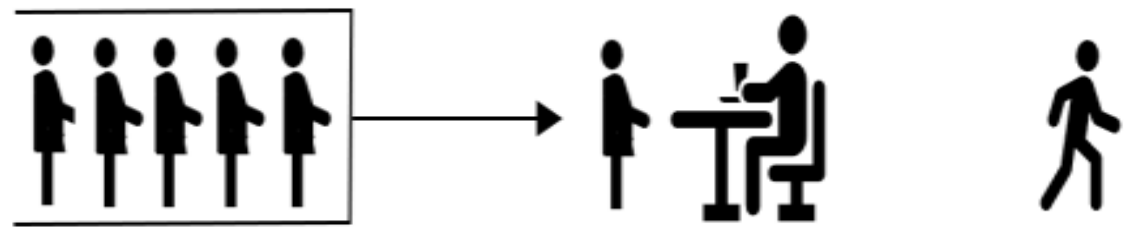

شكل رقم (11) صف واحد ومركزخدمة واحد

2. صفوف متعددة ومر اكز خدمة متعددة :

يمكن ملاحظة مثل هذه الحالة في مراكز الخدمة الكبيرة التي تتنوع فهها الخدمات أو تكثر الطلبات حيث يتم تخصيص عدد من المراكز ، وينتظم طالبوا الخدمة في صفوف أمام كل مركز كما في شكل رقم (12) 


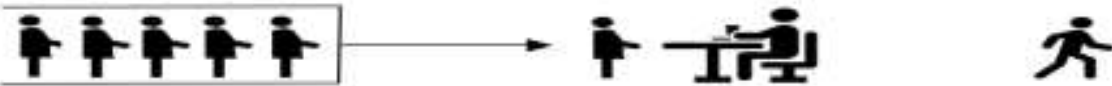

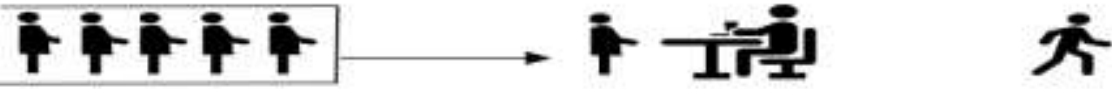

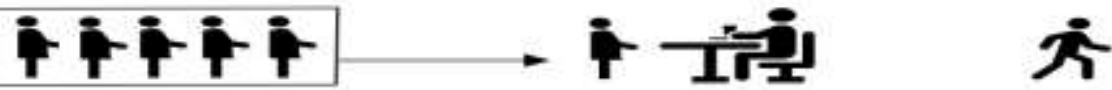

شكل رقم (12) صفوف ومر اكزخدمة متعددة

3. صفوف متعددة ومر اكز خدمة مرحلية متعددة :

هنا يتم تقديم الخدمة على مراحل / بمعنى أنه يلزم القيام بعدة إجراءات حتى يمكن

اعتبار الخدمة مكتملة لذا يتم تحدد مراكز خدمة متعددة تقدم فيها الخدمات بشكل مرحلي ( كما في حالة تنظيم جولة ارشادية في المكتبة للعملاء الجدد حيث يتعين التعرف مرف ماده على المصادر والخدمات والانتقال من مركز خدمة إلى آخر، شكل رقهم رقهم (13)

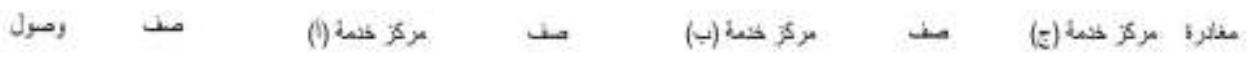

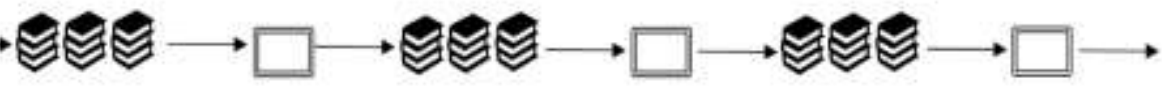

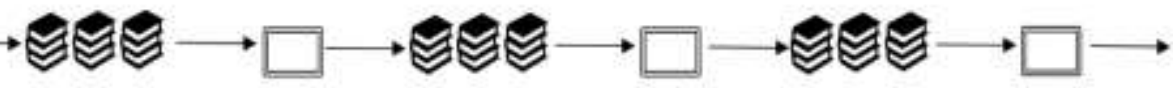

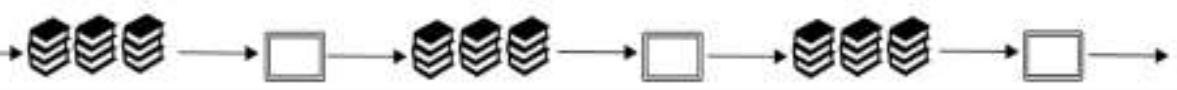

شكل رقم (13) صفوف ومر اكزخدمة متعددة

4. - صفوف متعددة ومركز خدمة مركزية :

في هذه الحالة وبالنظر إلى طبيعة الخدمة المطلوبة يتم تخصيص مركز خدمة

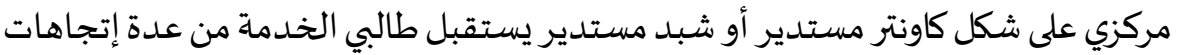

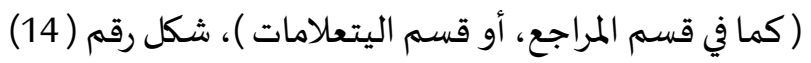




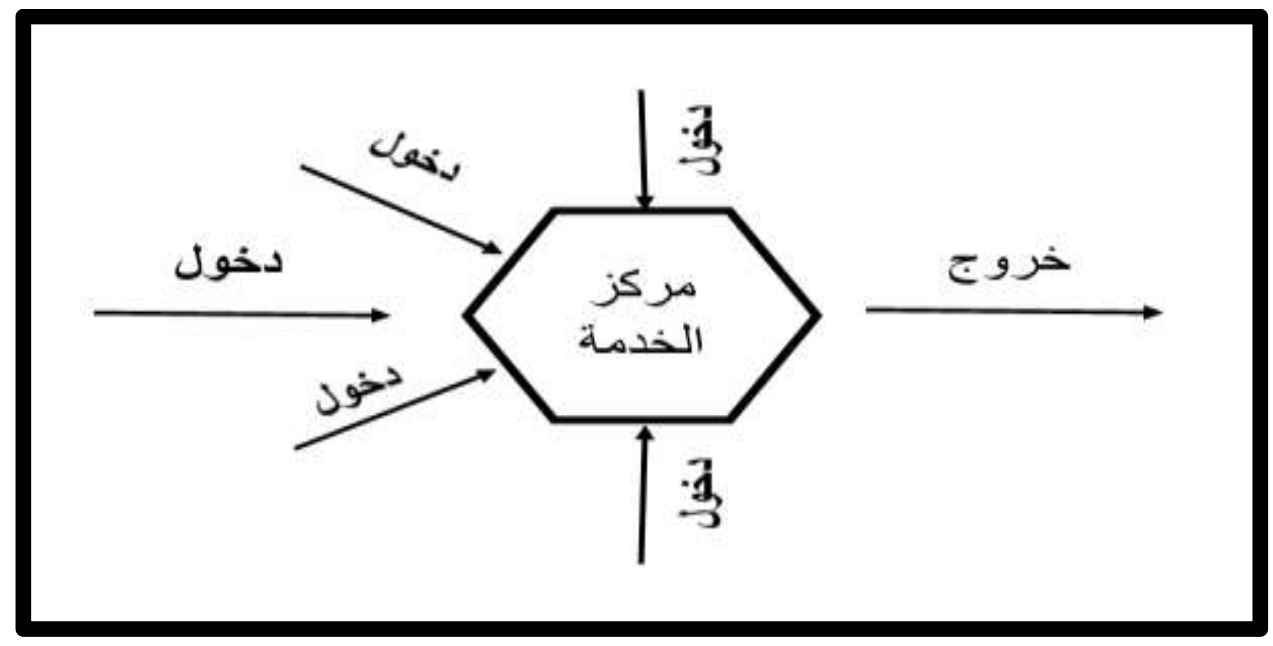

$$
\text { شكل رقم (14) صفوف متعددة ومركز خدمة مركزية }
$$

تطبيقات نظرية الصفوف :

هناك تطبيقات كثيرة لنظرية الصفوف في مجالات عديدة إلا أنه يلاحظ محدودية

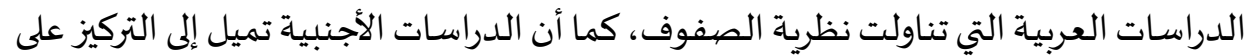

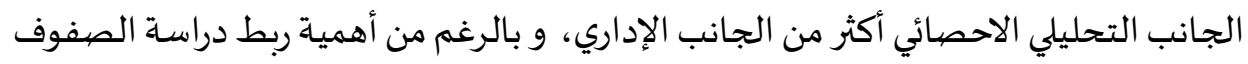

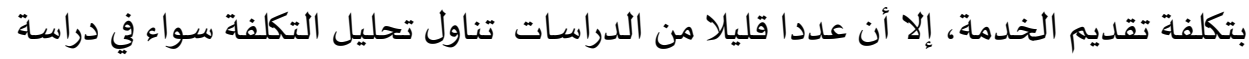

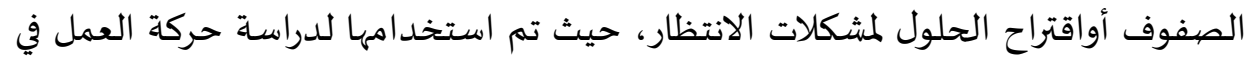

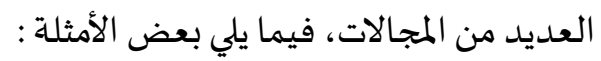

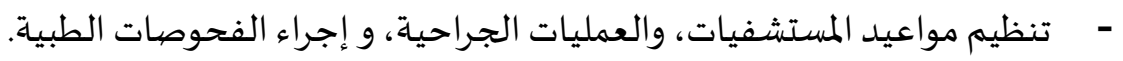

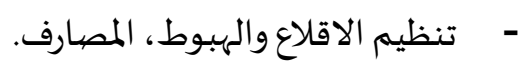

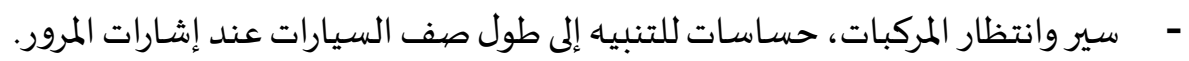

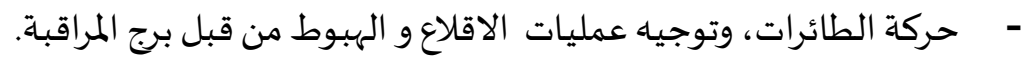

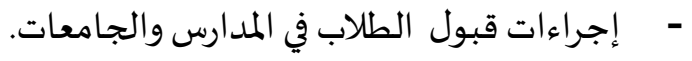

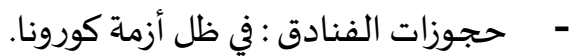

يوضح الجدول رقم ( 3 ) أمثلة للخدمات المكتبية والوحدات ألمورنات المسئولة عن تقديمها. يمكن

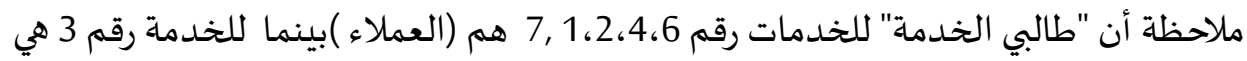


(السيارات المخصصة لتقديم خدمات المكتبة المتنقلة) التي تجوب الأحياءو الضيواحي، بينما الوحدات طالبة الخدمة في رقم 7 هي (الكتب المعارة). جدول رقم (3) أمثلة توظيف الصفوف في المكتبة ومركز المعلومات

\begin{tabular}{|c|c|c|}
\hline الخدمة المطلوبة & وحدة الخدمة & رمز الخدمة \\
\hline جولة في المكتبة & الارشاد & 1 \\
\hline الفهرسة والتصنيف & الاجراءات الفنية & 2 \\
\hline الاطلاع والاعارة & المكتبة المتنقلة & 3 \\
\hline حجز كتاب & كاونتر الاعارة & 4 \\
\hline الأسئلة المرجعية & المراجع & 5 \\
\hline حجز خلوة دراسية & علاقات العملاء & 6 \\
\hline ترفيف الكتب & خدمات المستفيدين & 7 \\
\hline
\end{tabular}

أما بالنسبة للدراسات التي تناولت تطبيق نظرية الصفوف في مجال المكتبات والمعلومات فهي محدودة جدا وتكاد تكون الدراسات العربية نادرة، ويعد مورس (8) يعد أول من حاول

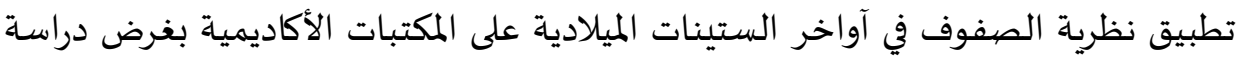
الصفوف التي تنشا أمام كاونتر الاعارة، تبعاه وارويك (9) في 1994 الذي حاول تقديم نموذج مطور لاستخدام النظرية لقياس مدة الاعارة، وفي دراسـة أخرى لله (10) عام 1998 حاول معاينة حجز أوعية المعلومات وربطها بزمن الانتظار، كما ناقش Acharaya و Ravidran (1999) تطبيق نظرية الصفوف في مجالات المكتبات والمعلومات خصوصيا في مجال إعارة المصادر وربط المبط رضا العميل بالزمن الذي يستغرقه للحصيول على المادة أو الخدمة المطلوبة ـ في الآونة الأخيرة Somvanshi

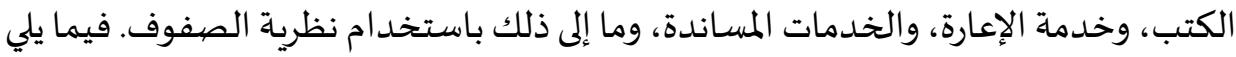
استعراض لبعض الدراسات : 
opac تناول Sampath تطبيق نظرية الصفوف في المكتبات مع التركيز على نظام

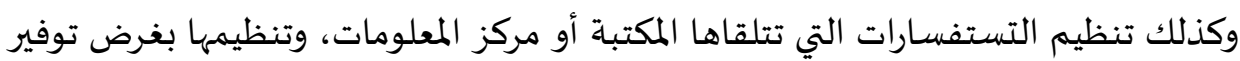

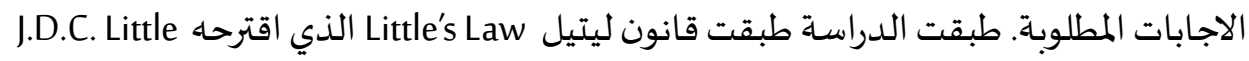
(14) عام 1961 والذي ينص على أن عدد العملاء في مركز الخدمة يساوي ناتج معدل وصهول

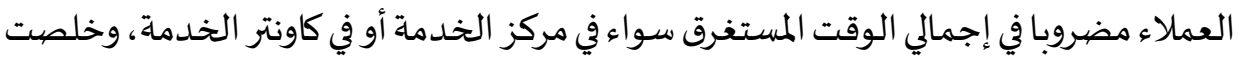

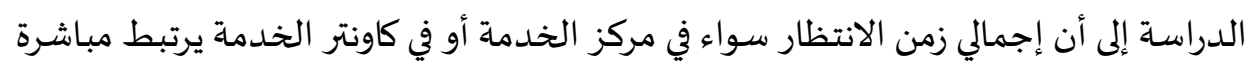

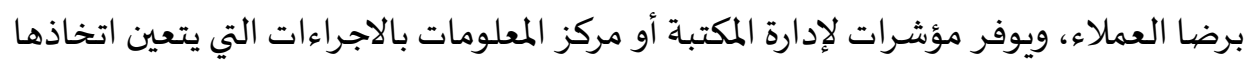

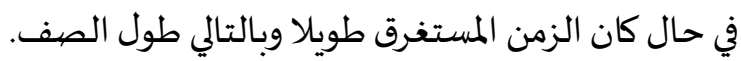

بينما اعتبرت دراسة LI Jing-Jing, LI Xi-Min أن إدارة المكتبات من الإدارات المعنية

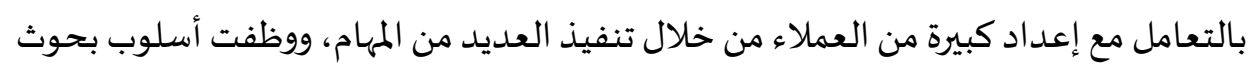
إدارة العمليات The Management Operation Research Method

ومثلت العمليات المكتبية في الظروف الاعتيادية من خلال 3 نماذج توضح ظروف عمل

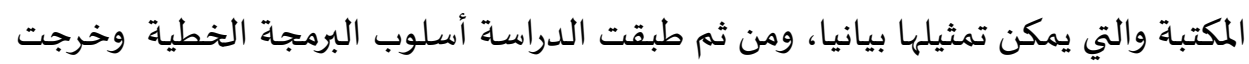

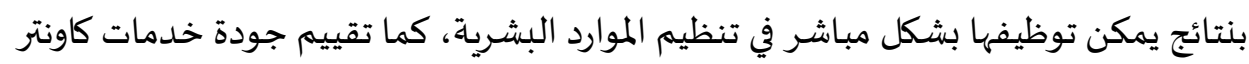

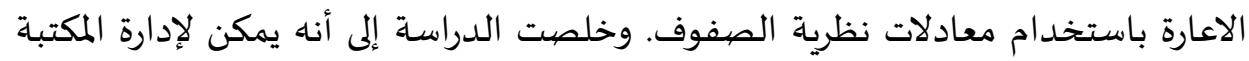

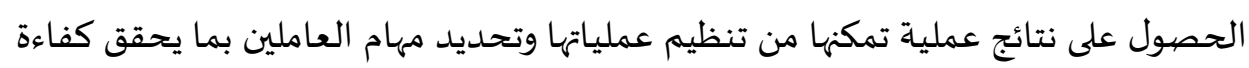
النشاط من جهة و رضا العملاء من جهة أخرى.

وهناك دراسة Warwick (16) التي هدفت إلى تشخيص تطبيق نظرية الصفوف في

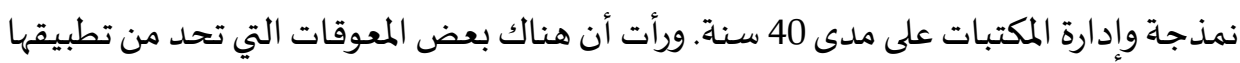

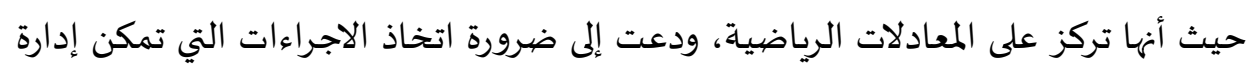

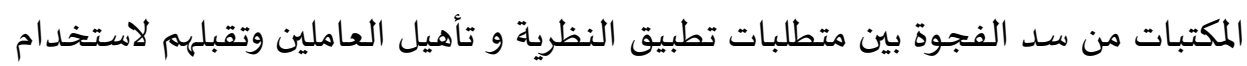

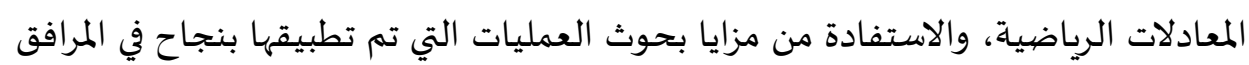
الأخرى. 
أما Srindhar (17) فقد تناول الطرق الكمية التي رأت أنه تم تجاهلها من قبل المكتبيين،

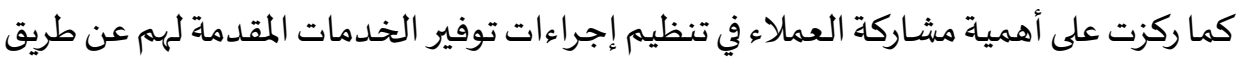

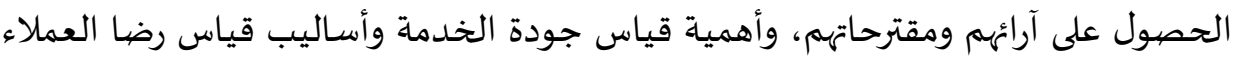

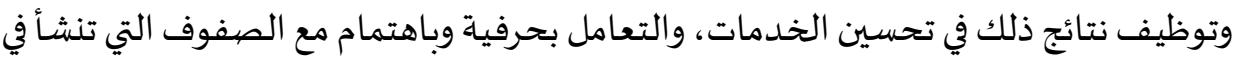
المكتبات ومراكز الخدمات أخذا في الاعتبار العوامل النفسية التي تؤثر على رضيا العماء العملاء

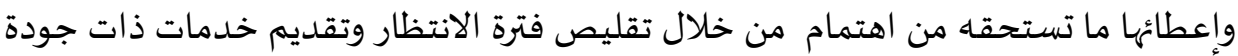

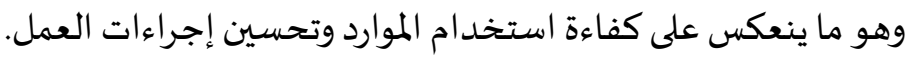

وخلصت الدراسة إلى مجموعة من المتوصيات المفيدة منها الحاجة إلى ربط العمليات

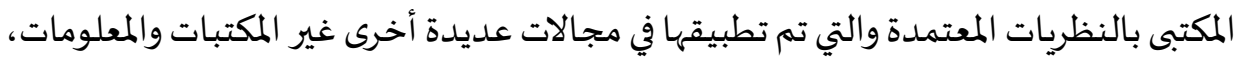

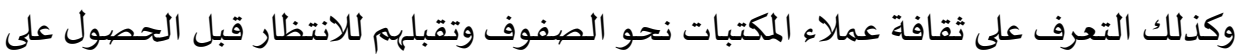

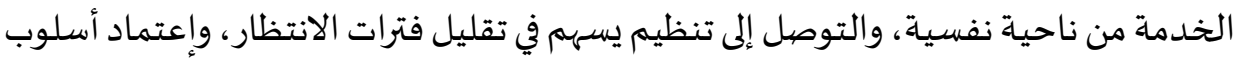
الحجز المسبق بحيث يتم تقديم الخدمات وفقا لمواعيد الحجز، وإضفاء بعض العضه العناصر التي تسهم في الحد من الملل، وتساعد على استثمار وقت الانتظار بما يعود بالفائدة. ومن الدراسات التي تناولت نظرية الصفوف في مجالا أخرى ما يلي :

دراسة غلاب (18) التي هدفت إلى التعرف على دور نظرية الصفوف في إتخاذ أفضل

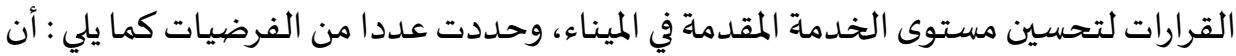

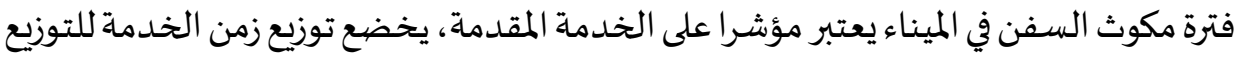

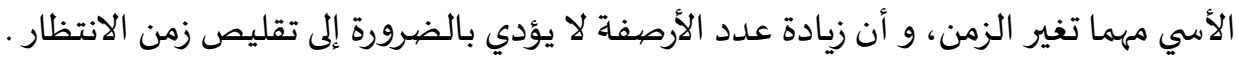

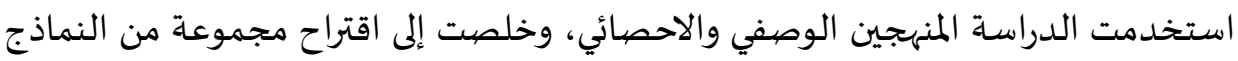

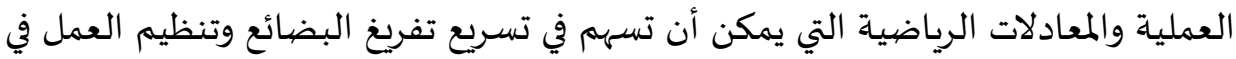
الميناء.

تناولت دراسة , Sabo, and Oyefabi (19) تنظيم خدمة المرضى في العيادات الخارجية حيث وظفت المنهج المستي واستخدمت أداتي الاستبيان والملاحظة للتعرف على خصائص 210 مريض أثناء تواجدهم في العيادات والوقت المستغرق في الانتظار قبل الكشف عليهم من قبل فيل 
الأطباء، ومدى رضاهم عن الخدمة المقدمة من حيث زمن الانتظار كما تعرفت على مقترحاتهم،

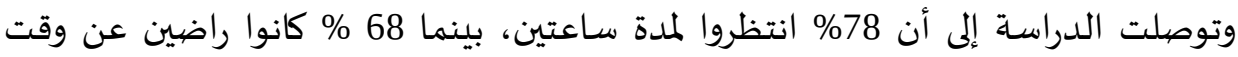
الانتظار،واقترحت زيادة عدد الأطباء في العيادات، كما اقترحت الحزم بشأن التزام الأطباء

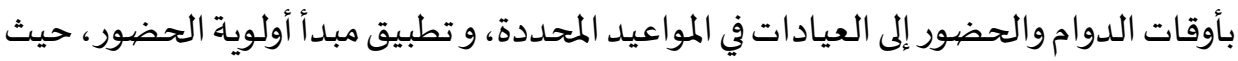
تضمن لاحظت الدراسـة تلقي العاملين لرشاوى لتقديم المرضى في الصف الصف على غيرهم. واقترحت الدراسة تفعيل آلية واضحة للحصول على التغذية الراجعة وتحليل مقترحات المرضى واتخاذ القرارات الكفيلة بتحقيق رضا المرضى. وتناول Nasir Uddin (20) وآخرون نظام إدارة صفوف الانتظار الآلي الذي نظام يساعد مزود الخدمة على إدارة الخدمات المقدمة بطريقة فعالة، واوضح المزايا العديدة التي يمكن

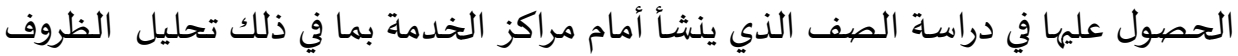
المرتبطة بطول الصف، وتقديم بيانات تفصيلية يتم على أساسها اتخاذ القرار المناسب أخذا في

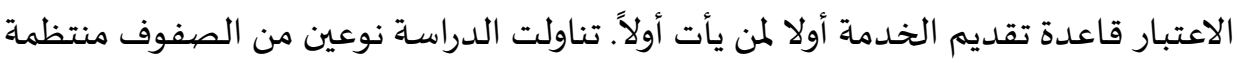

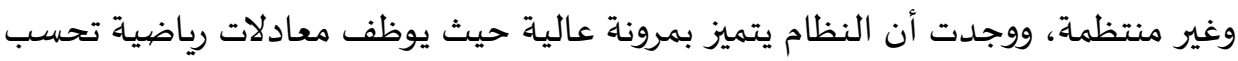

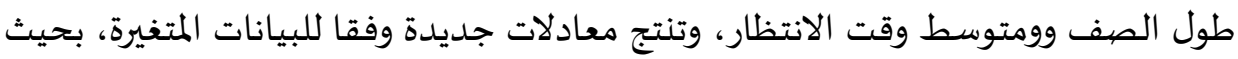

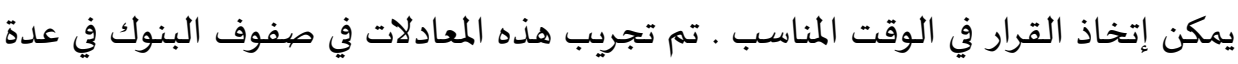

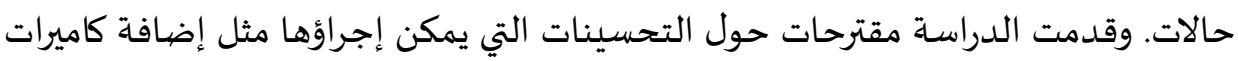

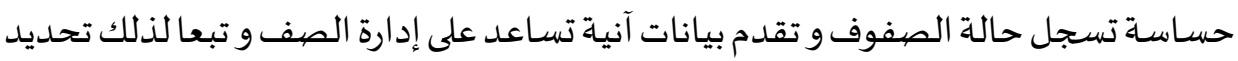
عدد نقاط تقديم الخدمات بزيادتها أو تقليصهيا.

أما دراسة , Siripongdee, Fongsuwan and Trimetsoontorn (21) فقد تناولت دراسـة صفوف الانتظار في 73 فرعا لمصلحة الكهرباء في تايلند التابعة للقطاع العام، و ربطت الدراسـة الخدمات المقدمة وطول فترة الانتظار ومدى استجابته لنمو قطاع الأعمال والصناعة فئاعة في البلاد.

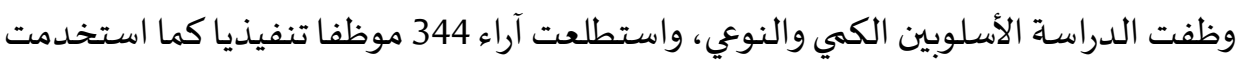

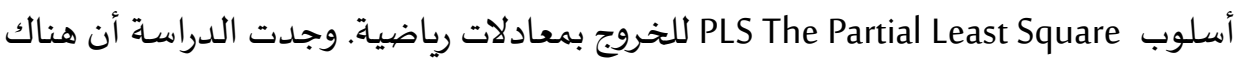

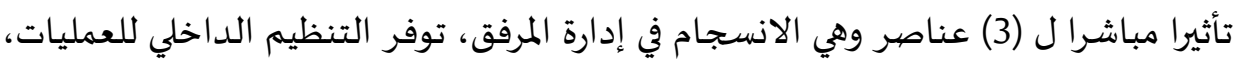


وتشجيع العاملين على تجويد العمل بمعنى أن العلقة طردية بين سلاسة ووضوح التنظيم

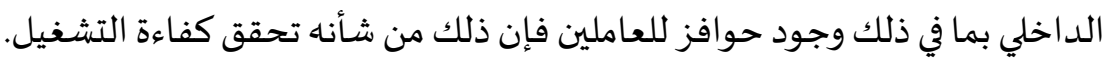

\section{نماذج من حزم البرامج الجاهزة للتعامل مع الصفوف :}

هناك العديد من التطبيقات التي تتيح تصهيم نموذج محاكاة لأنظمة مختلفة، ومنها نظرية الصفوف، حيث يمكن اختبار إجراءات تقديم الخدمة بما في ذلك تنظيم صفوف

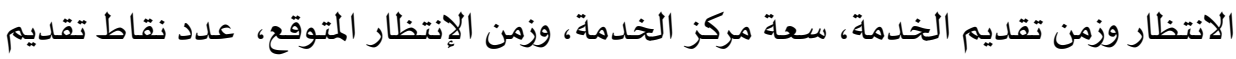

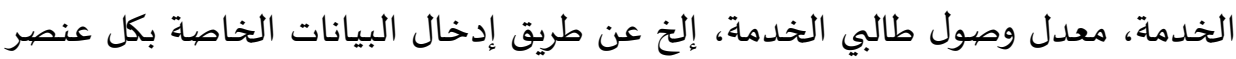

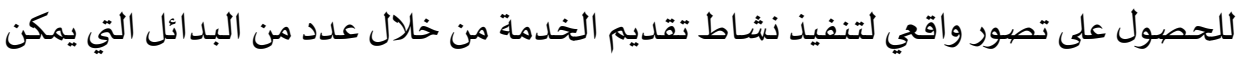
الاختيار من بينها بما يلئم ظروف كل مرفق. من امثلة على هذه البرامج تشمل التالي:

1. ANYLOGIC (https://www.anylogic.com)

2. MATLAB (https://www.mathworks.com/products/matlab.html)

3. SIMSCALE (https://www.simscale.com)

4. SIMUL8 (https://www.simul8.com)

5. COMSOL MULTIPHYSICS (https://www.comsol.com

6. SIMULINK (https://www.mathworks.com/products/simulink.html)

7. ARENA (http://info.arenasimulation.com/v16-esd-0)

مما سبق، يستنتج أنها يمكن توظيف نظرية الصفوف في مجالات كثيرة يتم توفير الخدمة فيها لعدد كبير من العملاء أخذا في الاعتبار ظروف نشوء الصفوف وطات وطاقة مراكز الخدمة. في

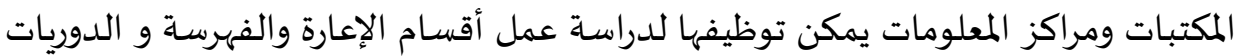
وقسم ترميم وصيانة أوعياة المعلومات ومعمل الحاسب الآلي وغيرها إلى جانب (قسم المراجع) والاستفادة من كم كبير من المعارف الصريحة والضمنية التي تتوفر في هذه الأقسام.

بغرض شرح المفاهيم السابقة تتناول الدراسة (قسم المراجع). على فرض أن القسم

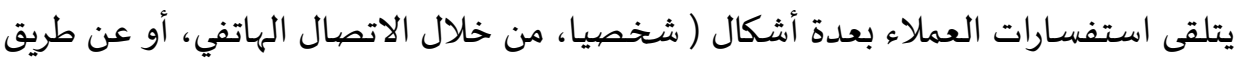


البريد الإلكتروني )، وجميع هذه الحالات يدخل الصف فيها عنصرا أساسيا. أخذا في الاعتبار أن أن التها

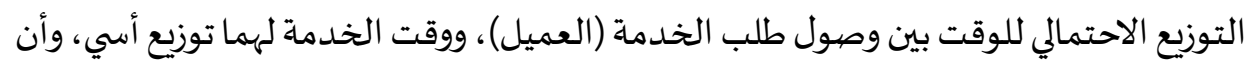

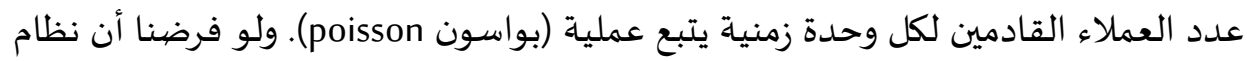

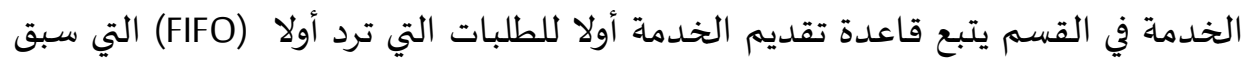
أيضاحها، فإنه يمكن نمذجة هذا النظام كما يلي :

Little's law was first introduced in a formal way by J. D. C. Little (1961) to solve problems in

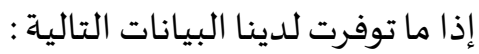

- أن متوسط عدد العملاء الذين يرتادون قسم المراجع (X) : 7 عملاء / الساعة

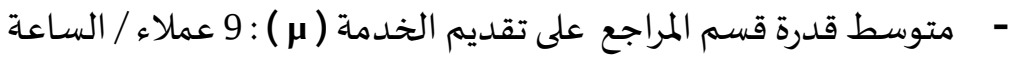

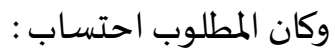
1. 1 احتمالية إشغال القسم

2. متوسط عدد العملاء في الصف "طول الصفئ الصفئ 3. العدد المتوقع للعملاء في القسم

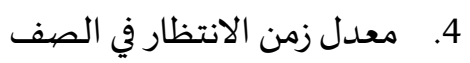

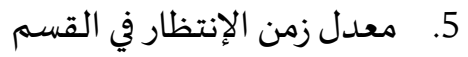
6. متوسط زمن تقديم الخدمة المرجعية

لتوفير المعلومات السابقة يمكن تطبيق المعادلات الرياضية التالية :

$$
\begin{array}{rlr}
p & =\frac{\lambda}{\mu} & \text { معادلة رق (1) معادلة رقم (2) معادلة رقم (3) } \\
\mathrm{Lq} & =\frac{p^{2}}{1-p} & \text { مع رقلة } \\
\mathrm{Ls} & =\frac{p}{1-p} & \text { (4) }
\end{array}
$$




$$
\begin{aligned}
& \text { Ws }=\frac{1}{\mu-\lambda} \quad \text { معادلة رقم (5) } \\
& \text { St }
\end{aligned}
$$

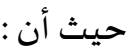

$$
\begin{aligned}
& \text { " = " معدل قدرة القسم على أداء الخدمة " } \\
& \text { " = " معدل وصيول العملاء } \\
& \text { " = p } \\
& \text { " = Ls } \\
& \text { "متوسط عدد العملاء في الصف ( طول الصف )" Lq } \\
& \text { "معدل الانتظار في القسم ( من وقت الوصيول إلى المغادرة )" Ws } \\
& \text { " =Wq } \\
& \text { " متوسط وقت الخدمة " St } \\
& \text { أولا : احتمالية إشغال القسم : }
\end{aligned}
$$

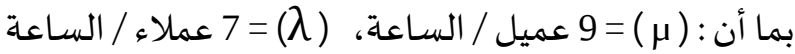

$$
\begin{aligned}
& \text { فإن إحتمالية إشـال قسم المراجع يمكن حسـابها كالتالي: } \\
& \mathrm{p}=\frac{\lambda}{\mu}=\frac{7}{9}=0.778=\% 78.8
\end{aligned}
$$

أي أن نسبة اشغال قسم المراجع هي.788\%، بمعنى أن 21.2\% من طاقة القسم شـاغرة، وتعتبر طاقة غير مستغلة.ووفي هذه الحالة قد تجد الإدارة أن نسبة الطاقة غير المستغلة مناسبة لمواجهة أي طارئ أو زيادة مفاجئة في عدد العملاء أو في عدد الاستفسارات المرجعية، مما يعني الابقاء على العاملين في القسم بنفس العدد. بالمقابل، فإن النسبة العالية للطاقة غير المستغلة قد تعبر عن خلل أو خسارة أو فقد، وقد تكون نتيجة توظيف عدد الموظفين يفوق الحاجة الفعلية، كما قد يعني سوء توزيع المهام الموكلة إليهم أو خلل في ساعات العمل، إلخ. 
هنا، فإنها بناء على المعطيات المتاحة والمعرفة الضيمنية المتوفرة نتيجة معايشة الوضع

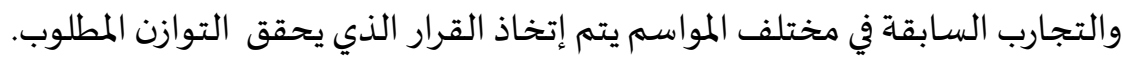

ثانيا : متوسط عدد العملاء في الصف "طول الصف" :

$$
\begin{aligned}
& \text { p = حيث أن P } \\
& \text { Lq }=\frac{p^{2}}{1-p} \quad \\
& \mathrm{Lq}=\frac{0.78^{2}}{1-0.78}=\frac{0.61}{0.22}=2.77 \approx 3 \text { عملاء } \\
& \text { ثالثا : العدد المتوقع للعملاء في القسم : } \\
& \text { حيث أن } 78 \text { حيث } \\
& \text { Ls }=\frac{p}{1-p} \text { فإن } \\
& \text { Ls }=\frac{0.78}{1-0.78}=3.55 \approx 4 \text { عمل } \\
& \text { رابعا : معدل زمن الانتظارفي الصف : }
\end{aligned}
$$

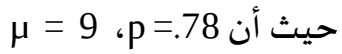

$$
\begin{aligned}
& \text { فq }
\end{aligned}
$$

دو

خامسا : معدل زمن الإنتظارفي القسم : - (

$$
\begin{aligned}
& \lambda=7 \text { ، حيث أن } 9=9 \\
& \text { Ws }=\frac{1}{\mu-\lambda}
\end{aligned}
$$

Ws $=\frac{1}{9-7}=0.50$ دقيقة 30 = سـاعة 


\section{سادسا : متوسط زمن تقديم الخدمة المرجعية :}

$$
\begin{aligned}
& \text { حيث أن Ws= 30, Wq=23.6 } \\
& \text { فt }
\end{aligned}
$$

St

الشكل رقم (15) يوضح نتيجة المعادلة السابقة التي تعبر عن الزمن الذي يستغرقه تقديم الخدمة المرجعية لعميل واحد.

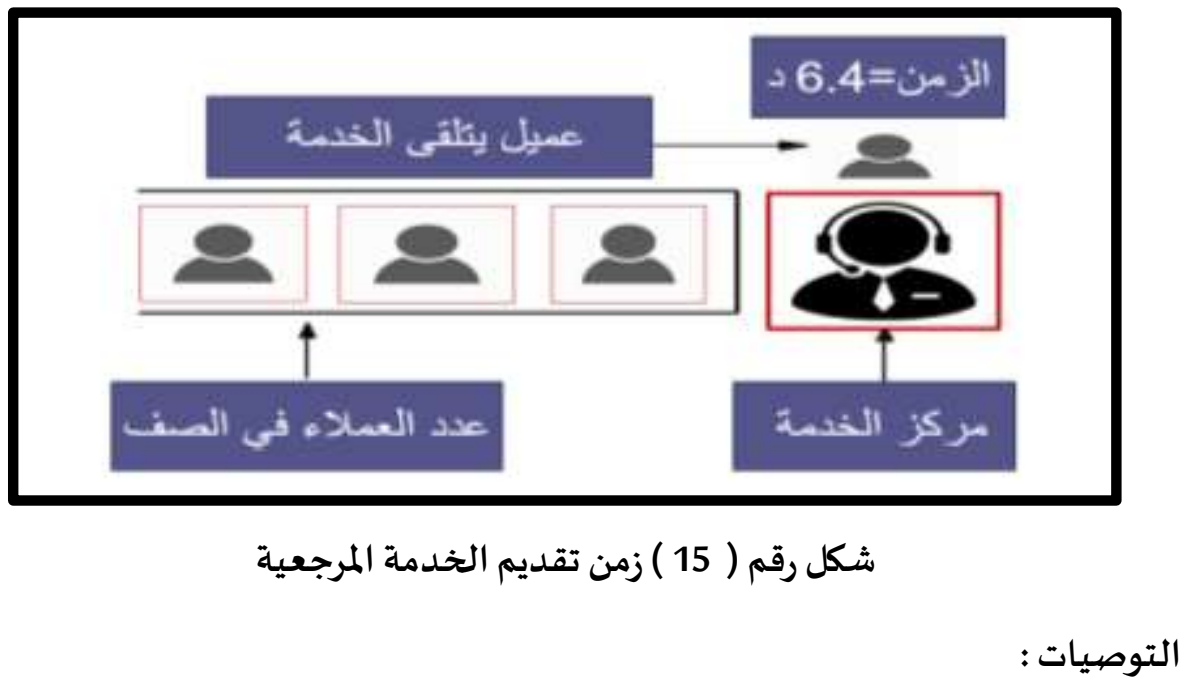

بناء على ما تمت مناقشته أعلاه تطرح الدراسة التوصيات التالية :

1. الاهتمام بالمعرفة الضمنية المتمثلة في خبرات وتجارب أخصائي المكتبات ومراكز

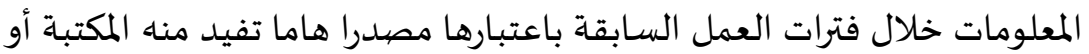
مركز المعلومات في تطبيق نظرية الصفوف. 2. ينبغي عدم اغفال المتغيرات التي يصعب ترجمتها عدديا بالرغم من تأثيرها بشكل

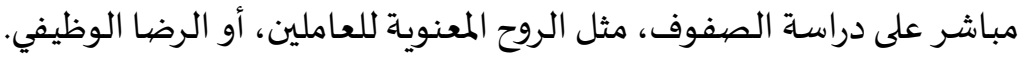


3. الاهتمام بالجوانب السلوكية والانسانية سواء بالنسبة للعاملين أو العملاء ضمن

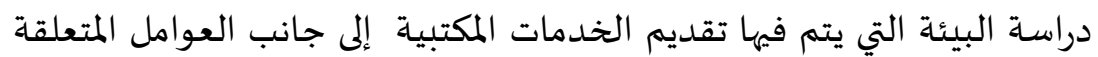

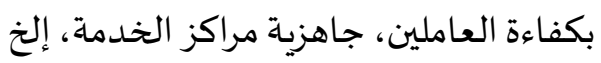
4. أن تكون نمذجة الحالة بطريقة توفر المعرفة الكافية التي تتيح تنفيذ المحاكاة. 5. ينبغي أن يكون تطبيق نظرية الصفوف لتنظيم ومتابعة العمل في المكتبة أو مركز

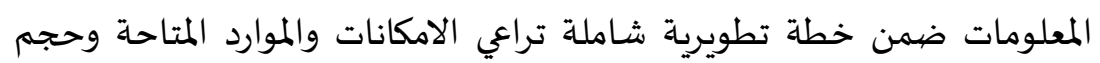
الطلب على الأقسام والوحدات. 6. أن تهتم المكتبة أو مركز المعلومات بالتوصيف الوفئ الوحيفي للعاملين المتضمن تحديد المهام والمسئوليات وتوضيح علاقة كل قسم بالأقسام الأخرى.

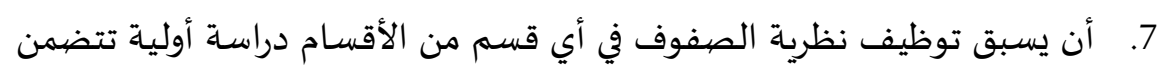
التعرف على أوقات الذروة، ونمط الطلبات في المواسم. 8. الاهتمام ببيئة العمل وتوفير العناصر الضرورية التي تحد من أثر الانتظار، بما في ذلك إعادة النظر في المساحات المخصصية لتقديم الخدمة المكتبية، الاهتمام بتخصيص مراكز خدمة لذوي الاحتياجات الخاصة.

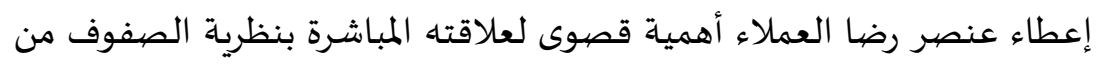

حيث زمن الانتظار، زمن تقديم الخدمة، أو كيفية تقديمها.

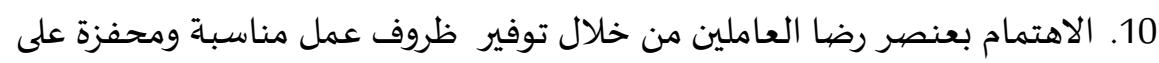
العطاء

11. الاهتمام بالجودة الشاملة التي تشكل دراسة الصفوف أحد روافدها المهمة.

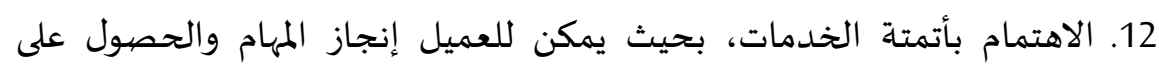

$$
\text { الخدمات دون الحاجة إلى الانتظار لفترات طويلة. }
$$

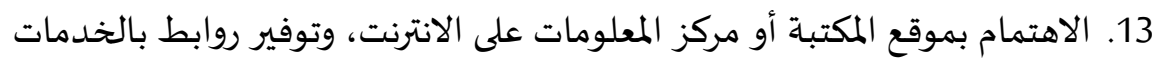

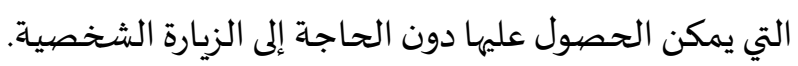

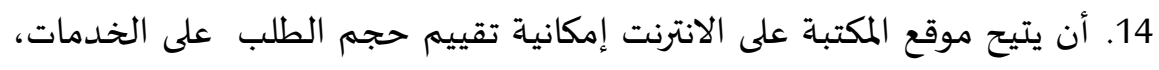

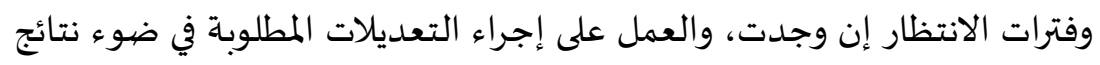

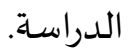


15. تشجيع العملاء على ابداء آرائهم ومقترحاتهم وفق آليات سهلة ومريحة لتلقي التغذية

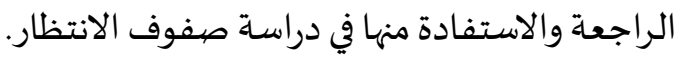

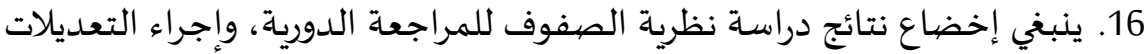
اللازمة كلما اقتضت الحاجة. 


\section{المراجع}

(1) Nasir Uddin, and others. "Automated Queue Management System". Global Journal of management and Business Research. V. 16, Issue, 2016

(2) Kappi, M. "Managing Modern Libraries: Challenges for Academic Libraries in the 21st Century". Nov 2018. Available at : https://www.researchgate.net/publication/337103156_Managing_Modern_L ibraries_Challenges_for_Academic_Libraries_in_the_21st_Century (Visited June 16 2021)

(3) Roknuzzaman, MD and Umemoto, K. " How Library Practitioners View Knowledge Management in Libraries: A Qualitative Study" . Library Management. V. 30 No. (8/9). Oct 2009,

(4) Robert D. Stueart and Barbara B. Moran Library and Information Center Management , 7TH Ed.2007. Available at : https://www.ebay.com/itm/185049495980?chn=ps\&mkevt=1\&mkcid=28 Visited June 17 2021)

(5) Abu-Rumman, A. “Gaining Competitive Advantage through Intellectual Capital and Knowledge Management: an Exploration of Inhibitors and Enablers in Jordanian Universities". Problems and Perspectives in Management. V. 16, Issue 3,2018

الصعيب، خالد " تحليل التكلفة والعائد "، 24 يناير 2018، متاح في

https://mafaheem.info/?p=434

(7) Law, A. and Kelton ,D. Simulation Models and Analysis. $2^{\text {nd }}$ ed. New york : McGraw·Hill, InC. 1991

(8) Morse , P. "Queues, Inventories, and Maintenance: The Analysis of Operational System with Variable Demand and Supply". Publications in Operations Research, No. 1, NewYork: wiley 1958 
(9) Warwick, J.P. "A Queueing Network Model for Book Circulation", Collection Management, 19(1-2), 69-80. 1994

(10) Warwick, J.P. "A Queueing Theory Model for Book Reservations and Circulation". Collection Management, 23(1-2), 125-137.1998

(11) Acharaya and Ravidran. "Application of Queuing Theory to Libraries" , 1999. Available at : https://drtc.isibang.ac.in/Idl/handle/1849/252 1 (Visited Nov 152021 )

(12) Somvanshi , T.V.S. "Application of Queuing Models in Effective Library Management", presented Conference: AIMT Lucknow Volume: V. 1 No. 1 Sep. 2012

(13) Sampath, M.I.G." Application of Queuing Theory to Library Management Systems with Special Reference to OPAC Searching". Conference paper Sep 2019, Available, at : https://www.researchgate.net/publication/33 (Visited Feb 02 2021)

(14) Little, J. D. C. "A Proof for the Queuing Formula: $L=\lambda w$." Operations Research, 9, 1961, 383-387.

(15) LI Jing-Jing, LI Xi-Min" Application of Operation Research in Library Management". Advances in Social Science, Education and Humanities Research, V. 290, 2018

(16) Warwick, Jon “On 40 Years of Queuing in Libraries". Library Review.V.58, Issue 1

(17) Sridhar, M. S. "Waiting Lines and Customer Satisfaction". SRELS Journal of Information Management. V. 38, No. 2, pp. 99-112, 2001 
(18) رشيد غلاب. " تحسين خدمات الموانئ باستخدام نماذج صفوف الانتظار : حالة المؤسسة المينائية لسكيكدة، رسالة ماجستير غير منشورة، كلية علوم التسيير والعلوم الاقتصادية،

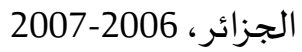

(19) Ameh, Nkeiruka, Sabo, B. , and Oyefabi , M. O.. "Application of Queuing Theory to Patient Satisfaction at a Tertiary Hospital in Nigeria", Niger Med I. Jan-Feb 2013. V. 54 No.164-67,

(20) Nasir Uddin, and Others. "Automated Queue Management System". Global Journal of Management and Business Research : a Administration and Management.. V. 16 Issue 1. 2016

(21) Surapong Siripongdee, Wanno Fongsuwan and Jirasek Trimetsoontorn,. "Structural Equation Modeling Analysis of Queuing System Performance at Thailand's Provincial Electricity Authority (PEA)". Research Journal of Business Management. V. 8. 57-69. 2014 